\title{
Ytterbium Acetate Promoted Asymmetric Reductive Amination: Significantly Enhanced Stereoselectivity
}

Thomas C. Nugent,* Mohamed El-Shazly, Vijay N. Wakchaure,

T. C. Nugent, Department of Chemistry, School of Engineering and Science, Jacobs

University Bremen (formerly International University Bremen), Campus Ring 1, 28759

Bremen, Germany, Fax: (+) 494212003229

t.nugent@jacobs-university.de

Table of Contents

1. Experimental section (general remarks).....S2

2. Synthesis of N-(S)- $\alpha$-MBA ketimine of 2-octanone.....S3

3. General procedure for stoichiometric asymmetric reductive amination with $\mathrm{Yb}(\mathrm{OAc})_{3}$ (enhanced de) .....S4

4. Specific examples for asymmetric reductive amination with stoichiometric $\mathrm{Yb}(\mathrm{OAc})_{3} \ldots . . \mathrm{S} 5$

5. General procedure for asymmetric reductive amination with catalytic $\mathrm{Yb}(\mathrm{OAc})_{3}$, $\mathrm{Y}(\mathrm{OAc})_{3}$, or $\mathrm{Ce}(\mathrm{OAc})_{3}($ normal de $) \ldots . . \mathrm{S} 10$

6. Specific examples for asymmetric reductive amination with catalytic $\mathrm{Yb}(\mathrm{OAc})_{3}$, $\mathrm{Y}(\mathrm{OAc})_{3}$, or $\mathrm{Ce}(\mathrm{OAc})_{3} \ldots . . . \mathrm{S} 11$

7. General procedure for asymmetric reductive amination with a Brønsted acid (normal de) ......S15

8. Specific examples of asymmetric reductive amination with acetic acid.....S15

9. Spectral data for synthesized compounds.....S17 


\section{Experimental Section}

\section{General Remarks}

NMR spectra were recorded on a JEOL ECX 400 spectrometer, operating at $400 \mathrm{MHz}$ $\left({ }^{1} \mathrm{H}\right)$ and $100 \mathrm{MHz}\left({ }^{13} \mathrm{C}\right)$ respectively. Chemical shifts $(\delta)$ were reported in parts per million $(\mathrm{ppm})$ downfield from TMS $(=0)$ or relative to $\mathrm{CHCl}_{3}(7.26 \mathrm{ppm})$ or $\mathrm{D}_{2} \mathrm{O}(4.79$

ppm) for ${ }^{1} \mathrm{H}$ NMR. For ${ }^{13} \mathrm{C}$ NMR, chemical shifts were reported in the scale relative to $\mathrm{CHCl}_{3}(77.0 \mathrm{ppm})$ or $\mathrm{D}_{2} \mathrm{O}\left(\mathrm{CH}_{3} \mathrm{OH}\right.$ internal reference, $\left.\delta=49.5 \mathrm{ppm}\right)$ as an internal reference. Multiplicities are abbreviated as: s, singlet; $d$, doublet; $t$, triplet; q, quartet; m, multiplet; br, broad. The coupling constants are expressed in Hz. FTIR spectra were obtained on Nicolet Avatar 370 spectrometer. Mass spectra were recorded on a Finnigan MAT 95 (EI) with an ionization potential of $70 \mathrm{eV}$. Elemental analyses were performed by an external vendor in Lindlar, Germany on an Elementar Vario EL III instrument. For amine products 2 , reaction progress and diastereomeric excess measurements were obtained using a Shimadzu GC-2010 instrument with a Rtx-5 amine column (Restec, 30 $\mathrm{m} \times 0.25 \mathrm{~mm}) ; \mathrm{T}_{\mathrm{inj}}=300{ }^{\circ} \mathrm{C}$ and $\mathrm{T}_{\text {det }}=300{ }^{\circ} \mathrm{C}$, and carrier gas He @ 24 psi were always constant. Program A: $50{ }^{\circ} \mathrm{C}(1 \mathrm{~min})$, then $14{ }^{\circ} \mathrm{C} / \mathrm{min}$ to $280{ }^{\circ} \mathrm{C}$ (hold $2 \mathrm{~min}$ ); Program B: $50{ }^{\circ} \mathrm{C}\left(1 \mathrm{~min}\right.$ ), then $14{ }^{\circ} \mathrm{C} / \mathrm{min}$ to $130{ }^{\circ} \mathrm{C}$ (hold $9 \mathrm{~min}$ ), then $20{ }^{\circ} \mathrm{C} / \mathrm{min}$ to $280{ }^{\circ} \mathrm{C}$ (hold 2 min); Program C: $50{ }^{\circ} \mathrm{C}(1 \mathrm{~min})$; then $14{ }^{\circ} \mathrm{C} / \mathrm{min}$ to $280{ }^{\circ} \mathrm{C}$ (hold $\left.1 \mathrm{~min}\right)$; Program D: 50 ${ }^{\circ} \mathrm{C}(1 \mathrm{~min})$; then $14{ }^{\circ} \mathrm{C} / \mathrm{min}$ to $280{ }^{\circ} \mathrm{C}$ (hold $5 \mathrm{~min}$ ). For hydrogenolyzed product primary amine 4d the enantiomeric excess of the trifluoroacetamide derivative was determined by gas chromatography using a Shimadzu GC-2010 instrument on a Chiraldex B-DP column (Astec, $30 \mathrm{~m} \times 0.25 \mathrm{~mm}$ ); $\mathrm{T}_{\mathrm{inj}}=200{ }^{\circ} \mathrm{C}, \mathrm{T}_{\mathrm{det}}=200{ }^{\circ} \mathrm{C}$, and carrier gas He @ 24 psi were constant. Program E: $130{ }^{\circ} \mathrm{C}(20 \mathrm{~min})$, then $20^{\circ} \mathrm{C} / \mathrm{min}$ to $180{ }^{\circ} \mathrm{C}$ (hold $\left.10 \mathrm{~min}\right)$, split ratio 
60:1. Column chromatography was performed using silica gel $60(0.040-0.063 \mathrm{~mm})$. Thin-layer chromatography (TLC) was performed using precoated plates of silica gel 60 $\mathrm{F}_{254}$ and visualized under ultraviolet irradiation $(254 \mathrm{~nm})$.

All reactions were performed under an inert atmosphere (nitrogen). All reagents were obtained from Sigma-Aldrich (except cyclohexyl methyl ketone, obtained from ABCR $\mathrm{GmbH} \& \mathrm{Co}$ ) and used without further purification. Before using the commercially purchased $\mathrm{Yb}(\mathrm{OAc})_{3}$ (Aldrich catalog number 544973, 99.999\% grade), $\mathrm{Y}(\mathrm{OAc})_{3}$ (Aldrich catalog number, 326046, 99.9\% grade), and $\mathrm{Ce}(\mathrm{OAc})_{3}$ (Aldrich catalog number, $529559,99.999 \%$ grade ), each was dried at $80{ }^{\circ} \mathrm{C}$ under high vacuum until a constant weight was achieved (12 h). The dried Lewis acids could be stored in dry screw cap glass bottles at room temperature, and these containers could be repeatedly opened to the atmosphere (at least 6 times without detrimental effect) without special precaution or need for a glovebox. In this way constant and repeatable results were always observed. The (S)- $\alpha$-methylbenzylamine (Aldrich catalog number, 115568) was of $98 \%$ chemical purity and 98\% ee. The Raney-Nickel (in water) was purchased from Fluka (Catalog number, 83440). $\mathrm{Pd}(\mathrm{OH})_{2} / \mathrm{C}[\leq 50 \%$ water, 20 wt \% loading (dry basis) $]$ was purchased from Aldrich (catalog number, 212911). Pt/C (1-4\% water, 5 wt \% loading) was purchased from Aldrich (Catalog number, 205931).

\section{Experimental Section}

\section{Synthesis of N-(S)- $\alpha$-MBA ketimine of 2-octanone}

p-Toluene sulphonic acid $(2 \mathrm{~mol} \%, 80 \mathrm{mg})$ was added to a double neck $100 \mathrm{~mL}$ round bottom flask, $60 \mathrm{~mL}$ of toluene was added, 2-octanone (22 mmol, 1.00 equiv, $3.45 \mathrm{~mL}$ ), and (S)- $\alpha$-methylbenzylamine (24.2 mmol, 1.10 equiv, $3.08 \mathrm{~mL})$ were added to the flask. 
The flask was connected to a Dean-Stark trap which was connected to a refluxing condenser. The mixture was refluxed for $24 \mathrm{~h}$ at $120{ }^{\circ} \mathrm{C}$. The mixture was allowed to cool and then the toluene was evaporated under vacuum. The residue was dissolved in hexane $(\sim 30 \mathrm{~mL})$, the solution was passed through filter paper and into a separatory funnel. Aqueous $\mathrm{NaHCO}_{3}(1.0 \mathrm{M}, 40 \mathrm{~mL})$ was added and after very brief mixing, the hexane layer was separated, washed with brine, dried over $\mathrm{MgSO}_{4}$ and filtered. The organic layer was concentrated under rotary evaporation then under high vacuum at $40{ }^{\circ} \mathrm{C}$ (with stirring) for $24 \mathrm{~h}(3.7 \mathrm{~g}, 57 \%$ yield $)$. GC analysis showed 96 area $\%$ of the imine and 4 area $\%$ of the starting ketone and amine. This imine was used for the further experiments.

\section{General procedure for the reduction of the N-(S)- $\alpha$-MBA ketimine of 2-octanone}

The imine (2.0 mmol, $462 \mathrm{mg}$ ) was added to a hydrogenation vessel, and then anhydrous $\mathrm{MeOH}(2.5 \mathrm{~mL})$ or THF $(2.5 \mathrm{~mL})$ was added. Dried $\mathrm{Yb}(\mathrm{OAc})_{3}$ or $\mathrm{Yb}(\mathrm{OTf})_{3}$ was then added, and the mixture was stirred for 30 min. A THF slurry of Raney-Ni (100 wt \% based on the imine, pre-triturated with EtOH $(\times 3)$ and then with anhydrous THF $(\times 3)$ before addition) (final reaction molarity $0.4 \mathrm{M}$ ) was then added and the reaction vessel pressurized at 120 psi (8.3 bar) of hydrogen. GC samples were worked-up using $\mathrm{NaHCO}_{3} /$ EtOAc.

\section{General procedure: Stoichiometric $\mathbf{Y b}(\mathrm{OAc})_{3}$ (enhanced de)}

In a dry reaction vessel $\mathrm{Yb}(\mathrm{OAc})_{3}(0.96 \mathrm{~g}, 2.75 \mathrm{mmol}, 1.1 \mathrm{~g}$ equiv) was added and subsequently evacuated under high vacuum for $5 \mathrm{~min}$ before flooding with nitrogen, anhydrous $\mathrm{MeOH}(2.5 \mathrm{~mL}, 1.0 \mathrm{M})$ was then added. To this solution a prochiral ketone 1 (2.5 mmol, 1.0 equiv) and (S)- $\alpha$-methylbenzylamine (0.35 mL, $2.75 \mathrm{mmol}, 1.1$ equiv) were added and subsequently stirred at room temperature for 20-30 min. A THF slurry of Raney-Ni (100 wt \% based on the ketone, pre-triturated with $\mathrm{EtOH}(\times 3)$ and then with 
anhydrous THF $(\times 3)$ before addition) was transferred to the reaction mixture using 2.5 $\mathrm{mL}$ of anhydrous THF (final molarity of reaction solution is $0.5 \mathrm{M}$ ) and the reaction vessel pressurized at $120 \mathrm{psi}(8.3 \mathrm{bar})$ of hydrogen. After $12 \mathrm{~h}$ at $22{ }^{\circ} \mathrm{C},<3$ area $\%$ of the ketone and imine intermediate remained, GC), and the reaction mixture was then diluted with $\mathrm{MeOH}(10 \mathrm{~mL})$, filter through a bed of celite, and the celite subsequently washed with $\mathrm{MeOH}(3 \times 10 \mathrm{~mL})$. The combined filtrate was then evaporated to dryness (rotary evaporate at $\leq 25{ }^{\circ} \mathrm{C}$ for short periods due to there semi-volatility), and $\mathrm{CHCl}_{3}(20 \mathrm{~mL})$ and aqueous $\mathrm{NaOH}(15 \mathrm{~mL}, 1.0 \mathrm{M})$ were added and this mixture stirred for $90 \mathrm{~min}$. After transferring to a separatory funnel, the $\mathrm{CHCl}_{3}$ layer was removed, and the aqueous layer further extracted with $\mathrm{CHCl}_{3}(3 \times 15 \mathrm{~mL})$. The combined $\mathrm{CHCl}_{3}$ extracts were filtered through a small bed of celite (removes turbidity) and the celite subsequently washed with $\mathrm{CHCl}_{3}(2 \times 15 \mathrm{~mL})$. The filtrate was then washed with saturated $\mathrm{NH}_{4} \mathrm{Cl}(2 \times 20 \mathrm{~mL})$ [removes residual $\alpha-\mathrm{MBA}$ ], then with brine $(1 \times 20 \mathrm{~mL})$, dried over $\mathrm{MgSO}_{4}$, filtered, and evaporated to dryness (rotary evaporate at $\leq 25{ }^{\circ} \mathrm{C}$ for short periods) to obtain the crude product (this material is used to determine the de). Purification by silica gel flash chromatography provides the mixture of diastereomers as a colorless viscous liquid (rotary evaporate at $\leq 25{ }^{\circ} \mathrm{C}$ for short periods), treatment with etheral $\mathrm{HCl}$ allows hydrochloride salt formation. Note that amine products $\mathbf{2}$ are considered semi-volatile and converted to $\mathrm{HCl}$ salts to enable their high vacuum drying (defined as $0.5-2.0 \mathrm{~mm} \mathrm{Hg}$ ) to constant weight (generally 12-24 h) for yield determination.

\section{(2S)-4-Methyl-N-((S)-1-phenylethyl)pentan-2-amine (2a)}

Reaction details: $\mathrm{Yb}(\mathrm{OAc})_{3}$ (1.1 equiv), 4-methyl-2-pentanone $(0.31 \mathrm{~mL}, 2.5 \mathrm{mmol}, 1.0$ equiv), (S)- $\alpha$-methylbenzylamine ( $0.35 \mathrm{~mL}, 2.75 \mathrm{mmol}, 1.1$ equiv). Reaction time: $12 \mathrm{~h}$; 94\% de. Purification by silica gel flash chromatography (hexanes/EtOAc/ $\mathrm{NH}_{4} \mathrm{OH}$, 
$83: 15: 2)$ gave the mixture of diastereomers as a colorless viscous liquid, which was then treated with etheral $\mathrm{HCl}$ to obtain the hydrochloride salt $(0.467 \mathrm{~g}, 78 \%$ yield $)$ after high vacuum drying. GC (program A, see: Experimental section (general remarks)): retention time [min]: major (S,S)-2a isomer, 10.9; minor (R,S)-2a isomer, 10.6. The NMR data of (S,S)-2a (free base) matches that reported in the literature. ${ }^{1}$

Major (S,S)-2a: ${ }^{1} \mathrm{H}$ NMR (400 MHz, $\left.\mathrm{CDCl}_{3}\right): \delta 7.36-7.20(\mathrm{~m}, 5 \mathrm{H}), 3.89(\mathrm{q}, \mathrm{J}=6.8 \mathrm{~Hz}$, $1 \mathrm{H}), 2.60-2.52(\mathrm{~m}, 1 \mathrm{H}), 1.63-1.56(\mathrm{~m}, 1 \mathrm{H}), 1.43-1.36(\mathrm{~m} 1 \mathrm{H}), 1.32(\mathrm{~d}, \mathrm{~J}=6.8 \mathrm{~Hz}, 3 \mathrm{H})$, 1.13-1.07 (m, 1H), $0.94(\mathrm{~d}, \mathrm{~J}=6.4 \mathrm{~Hz}, 3 \mathrm{H}), 0.89(\mathrm{~d}, \mathrm{~J}=6.8 \mathrm{~Hz}, 3 \mathrm{H}), 0.76(\mathrm{~d}, \mathrm{~J}=6.8 \mathrm{~Hz}$, $3 \mathrm{H}) .{ }^{13} \mathrm{C} \mathrm{NMR}\left(100 \mathrm{MHz}, \mathrm{CDCl}_{3}\right): \delta 146.5,128.3,126.7,126.5,55.2,48.4,46.6,25.1$, $24.4,23.6,22.3,21.6$.

\section{(2S)-4-Phenyl-N-((S)-1-phenylethyl)butan-2-amine (2c)}

Reaction details: $\mathrm{Yb}(\mathrm{OAc})_{3}$ (1.1 equiv), 4-phenyl-2-butanone $(0.37 \mathrm{~mL}, 2.5 \mathrm{mmol}, 1.0$ equiv), (S)- $\alpha$-methylbenzylamine ( $0.35 \mathrm{~mL}, 2.75 \mathrm{mmol}, 1.1$ equiv). Reaction time: $12 \mathrm{~h}$; $89 \%$ de. Purification by silica gel flash chromatography (hexanes/EtOAc $/ \mathrm{NH}_{4} \mathrm{OH}$, 78:20:2) gave the mixture of diastereomers as a colorless viscous liquid, which was then treated with etheral $\mathrm{HCl}$ to obtain the hydrochloride salt $(0.625 \mathrm{~g}, 87 \%$ yield) after high vacuum drying. GC (program D, see Experimental section (general remarks)): retention time [min]: major (S,S)-2c isomer, 15.5; minor (R,S)-2c isomer, 15.4. The NMR data of $(\mathrm{S}, \mathrm{S})-2 \mathrm{c}$ (free base) matches that reported in the literature. ${ }^{1}$

Major (S,S)-2c: ${ }^{1} \mathrm{H}$ NMR $\left(\mathrm{CDCl}_{3}, 400 \mathrm{MHz}\right): \delta 7.30-7.14(\mathrm{~m}, 10 \mathrm{H}), 3.85(\mathrm{q}, \mathrm{J}=6.4 \mathrm{~Hz}$, 1H), 2.69-2.49 (m, $3 \mathrm{H}), 1.85-1.83(\mathrm{~m}, 1 \mathrm{H}), 1.63-1.55(\mathrm{~m}, 1 \mathrm{H}), 1.29(\mathrm{~d}, \mathrm{~J}=6.4 \mathrm{~Hz}, 3 \mathrm{H})$, $1.02(\mathrm{~d}, \mathrm{~J}=6.4 \mathrm{~Hz}, 3 \mathrm{H}) .{ }^{13} \mathrm{C} \mathrm{NMR}\left(\mathrm{CDCl}_{3}, 100 \mathrm{MHz}\right): \delta 146.3,142.5,128.3,128.2$, $126.6,126.4,125.6,54.9,49.6,37.9,31.9,24.4,21.2$. 
Reaction details: $\mathrm{Yb}(\mathrm{OAc})_{3}(1.1$ equiv), 2-octanone (0.39 mL, $2.5 \mathrm{mmol}, 1.0$ equiv), $(S)$ a-methylbenzylamine (0.35 mL, $2.75 \mathrm{mmol}, 1.1$ equiv). Reaction time: $12 \mathrm{~h} ; 87 \%$ de. Purification by silica gel flash chromatography (hexanes/EtOAc/ $\mathrm{NH}_{4} \mathrm{OH}, 78: 20: 2$ ) gave the mixture of diastereomers as a colorless viscous liquid, which was then treated with etheral $\mathrm{HCl}$ to obtain the hydrochloride salt $(0.580 \mathrm{~g}, 86 \%$ yield $)$ after high vacuum drying. GC (program A, see Experimental section (general remarks)): retention time [min]: major (S,S)-2d isomer, 12.9; minor (R,S)-2d isomer, 13.1. The NMR data of $(\mathrm{S}, \mathrm{S})-2 \mathbf{d}$ (free base) matches that reported in the literature. ${ }^{1}$

Major (S,S)-2d: ${ }^{1} \mathrm{H}$ NMR (400 MHz, $\left.\mathrm{CDCl}_{3}\right): \delta$ 7.33-7.20 (m, 5H), $3.88(\mathrm{q}, \mathrm{J}=6.4 \mathrm{~Hz}$, 1H), 2.53-2.46 (m, 1H), 1.34-1.20 (m, 14H), $0.94(\mathrm{~d}, \mathrm{~J}=6.4 \mathrm{~Hz}, 3 \mathrm{H}), 0.88(\mathrm{t}, \mathrm{J}=6.4 \mathrm{~Hz}$, $3 \mathrm{H}) .{ }^{13} \mathrm{C} \mathrm{NMR}\left(100 \mathrm{MHz}, \mathrm{CDCl}_{3}\right): \delta 146.5,128.3,126.6,126.5,55.1,50.1,36.4,31.8$, $29.5,25.7,24.6,22.6,21.3,14.1$.

\section{(2S)-N-((S)-1-Phenylethyl)hexan-2-amine (2e)}

Reaction details: $\mathrm{Yb}(\mathrm{OAc})_{3}(1.1$ equiv), 2-hexanone $(0.31 \mathrm{~mL}, 2.5 \mathrm{mmol}, 1.0$ equiv), $(S)$ $\alpha$-methylbenzylamine $(0.35 \mathrm{~mL}, 2.75 \mathrm{mmol}, 1.1$ equiv). Reaction time: $12 \mathrm{~h} ; 85 \%$ de. Purification by silica gel flash chromatography (hexanes/EtOAc/ $\mathrm{NH}_{4} \mathrm{OH}, 88: 8: 2$ ) gave the mixture of diastereomers as a colorless viscous liquid, which was then treated with etheral $\mathrm{HCl}$ to obtain the hydrochloride salt $(0.480 \mathrm{~g}, 80 \%$ yield $)$ after high vacuum drying. GC (program A, see Experimental section (general remarks)): retention time [min]: major $(\mathrm{S}, \mathrm{S})-2 \mathrm{e}$ isomer, 11.3; minor $(\mathrm{R}, \mathrm{S})-2 \mathbf{e}$ isomer, 11.1. The NMR data of $(\mathrm{S}, \mathrm{S})$ $\mathbf{2 e}$ (free base) matches that reported in the literature. ${ }^{2}$

Major (S,S)-2e: ${ }^{1} \mathrm{H}$ NMR (CDCl3, $\left.400 \mathrm{MHz}\right): \delta$ 7.33-7.19 (m, 5H), $3.88(\mathrm{q}, \mathrm{J}=6.5 \mathrm{~Hz}$, 1H), 2.51-2.45 (m, 1H), 1.52-1.46 (m, 1H), $1.32(\mathrm{~d}, \mathrm{~J}=6.5 \mathrm{~Hz}, 3 \mathrm{H}), 1.28-1.15(\mathrm{~m}, 6 \mathrm{H})$, 
$0.94(\mathrm{~d}, \mathrm{~J}=6.34 \mathrm{~Hz}, 3 \mathrm{H}), 0.88(\mathrm{t}, \mathrm{J}=6.95 \mathrm{~Hz}, 3 \mathrm{H}) \cdot{ }^{13} \mathrm{C} \mathrm{NMR}(\mathrm{CDCl}, 100 \mathrm{MHz}): \delta$ $146.4,128.3,126.6,126.5,55.1,50.1,36.0,27.9,24.6,22.9,21.3,14.1$

\section{(2S)-N-((S)-1-Phenylethyl)butan-2-amine (2f)}

Reaction details: $\mathrm{Yb}(\mathrm{OAc})_{3}$ (1.1 equiv), 2-butanone ( $0.22 \mathrm{~mL}, 2.5 \mathrm{mmol}, 1.0$ equiv), (S)$\alpha$-methylbenzylamine (0.35 mL, $2.75 \mathrm{mmol}, 1.1$ equiv). Reaction time: $12 \mathrm{~h} ; 79 \% \mathrm{de}$. After stopping the hydrogenation, further $\mathrm{MeOH}$ was added, and this heterogeneous solution was filtered to remove the Raney-Ni, and excess ethereal $\mathrm{HCl}$ was added. This was concentrated to dryness (rotary evaporation) and then aqueous $\mathrm{HCl}(2.0 \mathrm{M})$ and ether were added. The acidic aqueous layer was removed and the $\mathrm{Et}_{2} \mathrm{O}$ was extracted with further extracted with aqueous $\mathrm{HCl}(2.0 \mathrm{M}, 2 \times 15 \mathrm{~mL})$. The aqueous acidic layer was basified with $\mathrm{NaOH}(4.0 \mathrm{M})$ to a $\mathrm{pH}=12-14$ and the free amine extracted with $\mathrm{CH}_{2} \mathrm{Cl}_{2}(4$ x $20 \mathrm{~mL}$ ). The combined organic extracts were dried over $\mathrm{Na}_{2} \mathrm{SO}_{4}$, filtered and to the filtrate ethereal $\mathrm{HCl}(2.0 \mathrm{M}, 4.0 \mathrm{~mL})$ was added. This solution was concentrated on rotary evaporator and after high vacuum drying ( $\geq 24 \mathrm{~h})$ afforded the $\mathrm{HCl}$ salt $(0.42 \mathrm{~g}, 79 \%$ yield) after high vacuum drying. GC (program B, see Experimental section (general remarks)): retention time [min] for the free base: major (S,S)-2f isomer, 13.0; minor $(\mathrm{R}, \mathrm{S})-\mathbf{2 f}$ isomer, 12.7. The NMR data of (S,S)-2f (free base) matches that reported in the literature. $^{2}$

Major (S,S)-2f: ${ }^{1} \mathrm{H}$ NMR (CDCl3, $400 \mathrm{MHz}$ ): $\delta$ 7.38-7.20 (m, 5H), 3.87 (q, J = $6.4 \mathrm{~Hz}$, 1H), 2.49-2.41 (m, 1H), 1.56-1.49 (m, 1H), 1.34-1.24 (m, $5 \mathrm{H}), 0.95(\mathrm{~d}, \mathrm{~J}=6.4 \mathrm{~Hz}, 3 \mathrm{H})$, $0.84(\mathrm{t}, \mathrm{J}=7.2 \mathrm{~Hz}, 3 \mathrm{H}) .{ }^{13} \mathrm{C} \mathrm{NMR}(\mathrm{CDCl} 3,100 \mathrm{MHz}): \delta 146.4,128.3,126.7,126.5,55.0$, $51.3,28.6,24.7,20.7,9.8$.

(2S)-3,3-Dimethyl-N-((S)-1-phenylethyl)butan-2-amine (2b) (Pt substrate) 
In a reaction vessel dry $\mathrm{Yb}(\mathrm{OAc})_{3}(0.96 \mathrm{~g}, 2.75 \mathrm{mmol}, 1.1 \mathrm{equiv})$ was added and subsequently evacuated under high vacuum for 5 min before flooding with nitrogen,, anhydrous $\mathrm{MeOH}(2.5 \mathrm{~mL}, 1.0 \mathrm{M})$ was then added. To this solution 3,3-Dimethyl-2butanone $(0.31 \mathrm{~mL}, \quad 2.5 \mathrm{mmol}, 1.0$ equiv $),(S)-\alpha$-methylbenzylamine $(0.35 \mathrm{~mL}, 2.75$ mmol, 1.1 equiv) were added. The reaction was then stirred at $50{ }^{\circ} \mathrm{C}$ for $2 \mathrm{~h}$. Pt-C $(98.0$ $\mathrm{mg}, 1.0 \mathrm{~mol} \%$ ) [The $98 \mathrm{mg}$ of $\mathrm{Pt} / \mathrm{C}$ was added in four equal portions, thus $24.5 \mathrm{mg}$ at $\mathrm{t}=$ $0 \mathrm{~h}, \mathrm{t}=2 \mathrm{~h}, \mathrm{t}=4 \mathrm{~h}$, and finally at $\mathrm{t}=6 \mathrm{~h}$ ] and THF $(2.5 \mathrm{~mL}$, final molarity of reaction vessel $0.5 \mathrm{M}$ ) was then added and the reaction vessel pressurized at 120 psi ( 8.3 bar) of hydrogen. The reaction was then stirred at $50^{\circ} \mathrm{C}$. After $22 \mathrm{~h}(<11$ area $\%$ of the ketone remained by $\mathrm{GC})$, the reaction mixture was diluted with $\mathrm{MeOH}(10 \mathrm{~mL})$, and the general procedure then followed to provide the crude product $(92 \%$ de). Purification by silica gel flash chromatography provided the mixture of diastereomers as a colorless viscous liquid (rotary evaporator at $\leq 25{ }^{\circ} \mathrm{C}$ for short periods), which was then treated with etheral $\mathrm{HCl}$ to obtain the hydrochloride salt $(0.395 \mathrm{~g}, 77 \%$ yield $)$ after high vacuum drying. GC (program A, see Experimental section (general remarks)): retention time [min]: major $(\mathrm{S}, \mathrm{S})-2 \mathbf{b}$ isomer, 10.9; minor $(\mathrm{R}, \mathrm{S})-2 \mathbf{b}$ isomer, 10.6. The NMR data of $(\mathrm{S}, \mathrm{S})-2 \mathbf{b}$ (free base) matches that reported in the literature. ${ }^{2}$

Major (S,S)-2k: ${ }^{1} \mathrm{H}$ NMR (400 MHz, $\left.\mathrm{CDCl}_{3}\right): \delta 7.35-7.20(\mathrm{~m}, 5 \mathrm{H}), 3.77(\mathrm{q}, \mathrm{J}=6.4 \mathrm{~Hz}$, 1H), 2.29 (q, J = 6.4 Hz, 1H), $1.27(\mathrm{~d}, \mathrm{~J}=6.4 \mathrm{~Hz}, 3 \mathrm{H}), 0.89-0.84(\mathrm{~m}, 12 \mathrm{H}) .{ }^{13} \mathrm{C} \mathrm{NMR}$ $\left(100 \mathrm{MHz} \mathrm{CDCl}_{3}\right): \delta 147.6,128.2,126.7,126.6,59.5,57.0,34.7,26.5,23.7,16.0$.

\section{(S)-2-aminooctane (4d)}

The diastereomeric amine mixture (2d) $(0.466 \mathrm{~g}, 2.0 \mathrm{mmol}, 86 \% \mathrm{de})$ was dissolved in $\mathrm{EtOH}(5.0 \mathrm{~mL}, 0.4 \mathrm{M})$ and hydrogenolysis was carried out in presence of $\mathrm{Pd}(\mathrm{OH})_{2} / \mathrm{C}$ $(0.196 \mathrm{~g}, 7.0 \mathrm{~mol} \%)$ at $8.3 \mathrm{bar}(120 \mathrm{psi})$ of hydrogen pressure at room temperature. After $10 \mathrm{~h}$, the catalyst was filtered through filter paper and which was subsequently washed 
with $\mathrm{EtOH}(2 \times 10 \mathrm{~mL}) .2 .0 \mathrm{M}$ etheral $\mathrm{HCl}(4.0 \mathrm{~mL})$ was then added to the filtrate, and this solution was evaporated to dryness to obtain an oil. The oil was triturated with hexane $(4 \times 10 \mathrm{~mL})$ and the residual hexane evaporated, this was repeated 3-4 times to obtain a white solid. Further drying for $15 \mathrm{~h}$ under high vacuum provided a white solid in qualitative purity $(0.25 \mathrm{~g}, 76 \%$ yield $)$. The trifluoroacetyl derivative of $\mathbf{4 d}$ had an ee of 85\% (chiral GC program E, see Experimental section (general remarks) and Supporting Information chromatograms). GC retention time [min]: major (S)-4d trifluoroacetamide isomer, 15.3; minor (R)-4d trifluoroacetamide isomer, 16.5 .

4d-HCl salt: ${ }^{1} \mathrm{H}$ NMR $\left(400 \mathrm{MHz}, \mathrm{CDCl}_{3}\right): \delta 8.32$ (br s, 3H), 3.32-3.29 (m, 1H), 1.82$1.56(\mathrm{~m}, 2 \mathrm{H}), 1.41-1.28(\mathrm{~m}, 11 \mathrm{H}), 0.87(\mathrm{t}, \mathrm{J}=6.4 \mathrm{~Hz}, 3 \mathrm{H}) .{ }^{13} \mathrm{C} \mathrm{NMR}\left(100 \mathrm{MHz}, \mathrm{CDCl}_{3}\right)$ : $\delta 46.9,40.2,31.8,29.4,26.3,23.9,22.6,14.0$.

4d-oxalate salt: The reported literature data for this compound is that of the oxalate salt for which the ${ }^{1} \mathrm{H}$ NMR is reported. We also formed this salt and found the ${ }^{1} \mathrm{H}$ NMR data for this oxalate salt of (S)-4d matched that reported: ${ }^{1} \mathrm{H}$ NMR $\left(400 \mathrm{MHz}, \mathrm{D}_{2} \mathrm{O}\right): \delta 3.32-$ $3.27(\mathrm{~m}, 1 \mathrm{H}), 1.64-1.47(\mathrm{~m}, 2 \mathrm{H}), 1.28-1.22(\mathrm{~m}, 11 \mathrm{H}), 0.81(\mathrm{t}, \mathrm{J}=6.4 \mathrm{~Hz}, 3 \mathrm{H}) .{ }^{3} \mathrm{We}$ additionally recorded the ${ }^{13} \mathrm{C}$ NMR $\left(100 \mathrm{MHz}, \mathrm{D}_{2} \mathrm{O}, \mathrm{CH}_{3} \mathrm{OH}\right.$ was used as the internal reference, $\delta=49.5 \mathrm{ppm}): \delta 164.9,48.6,34.7,31.5,28.8,25.2,22.5,18.3,14.0 .^{4}$

\section{General procedure: Catalytic $\mathrm{Yb}(\mathrm{OAc})_{3}, \mathrm{Y}(\mathrm{OAc})_{3}$, or $\mathrm{Ce}(\mathrm{OAc})_{3}$ (normal de)}

In a reaction vessel the Lewis acid $\left[\mathrm{Yb}(\mathrm{OAc})_{3} 10 \mathrm{~mol} \%, \mathrm{Y}(\mathrm{OAc})_{3}(15 \mathrm{~mol} \%)\right.$ or $\left.\mathrm{Ce}(\mathrm{OAc})_{3}(15 \mathrm{~mol} \%)\right]$ was added and subsequently evacuated under high vacuum for 5 min before flooding with nitrogen. To the vessel, anhydrous methanol $(2.5 \mathrm{~mL}, 1.0 \mathrm{M})$, ketone (2.5 mmol, 1.0 equiv), and $(S)$ - $\alpha$-methylbenzylamine $(0.35 \mathrm{~mL}, 2.75 \mathrm{mmol}, 1.1$ equiv) were added and stirred for $20-30 \mathrm{~min}$ at the temperature at which the hydrogenation was performed at $\left(22\right.$ or $\left.50{ }^{\circ} \mathrm{C}\right)$. A THF slurry of Raney-Ni (100 wt \% 
based on the ketone, pre-triturated with $\mathrm{EtOH}(\times 3)$ and then with anhydrous THF $(\times 3)$ before addition) was transferred to the reaction mixture using $2.5 \mathrm{~mL}$ of anhydrous THF (final molarity of reaction solution is $0.5 \mathrm{M}$ ). The vessel was then pressurized to the indicated pressure 120-290 psi (8-20 bar) of hydrogen and stirred at room temperature or at $50{ }^{\circ} \mathrm{C}$ as indicated. At $12 \mathrm{~h}(<3$ area $\%$ of ketone and intermediate imine by GC) the reaction mixture was worked-up as delineated in the section entitled: "General procedure: Stoichiometric $\mathrm{Yb}(\mathrm{OAc})_{3}$ (enhanced de).”

\section{(2S)-4-methyl-N-((S)-1-phenylethyl)pentan-2-amine (2a)}

Reaction details: $\mathrm{Yb}(\mathrm{OAc})_{3}(10 \mathrm{~mol} \%)$, 4-methyl-2-pentanone $(0.31 \mathrm{~mL}, 2.5 \mathrm{mmol}, 1.0$ equiv), (S)- $\alpha$-methylbenzylamine $(0.35 \mathrm{~mL}, 2.75 \mathrm{mmol}, 1.1$ equiv), pre-stirred $30 \mathrm{~min}$ at $50{ }^{\circ} \mathrm{C}$, and then hydrogenated at $50{ }^{\circ} \mathrm{C}$ and 120 psi (8.3 bar). Reaction time: $12 \mathrm{~h} ; 92 \%$ de. Purification by silica gel flash chromatography (Hexane/EtOAc/ $\mathrm{NH}_{4} \mathrm{OH}=87: 9: 4$ ) gave the mixture of diastereomers as viscous colorless oil, which then treated with etheral $\mathrm{HCl}$ to obtain the hydrochloride salt $(0.60 \mathrm{~g}, 79$ yield \%). GC (program D, see Experimental section (general remarks)) retention time [min]: major $(S, S)$-2a isomer, 11.8; minor $(R, S)$-2a isomer, 11.6, matched those reported in the literature. ${ }^{1}$

\section{(2S)-4-phenyl-N-((S)-1-phenylethyl)butan-2-amine (2c)}

Reaction details: $\mathrm{Yb}(\mathrm{OAc})_{3}(10 \mathrm{~mol} \%)$, 4-phenyl-2-butanone $(0.37 \mathrm{~mL}, 2.5 \mathrm{mmol}, 1.0$ equiv), (S)- $\alpha$-methylbenzylamine (0.35 $\mathrm{mL}, 2.75 \mathrm{mmol}, 1.1$ equiv), pre-stirred $30 \mathrm{~min}$ at room temperature; hydrogen pressure 8.3 bar (120 psi); hydrogenation was performed at room temperature; reaction time: $12 \mathrm{~h} ; 80 \%$ de. Purification by silica gel flash chromatography (Hexane/EtOAc/ $\left.\mathrm{NH}_{4} \mathrm{OH}=83: 15: 2\right)$ gave the mixture of diastereomers as viscous colorless oil, which then treated with etheral $\mathrm{HCl}$ to obtain the hydrochloride salt (0.63 g, 87 yield \%). GC (program D, see Experimental section (general remarks)) 
retention time [min]: major $(S, S)-2 \mathrm{c}$ isomer, 16.5 minor $(R, S)$-2c isomer, 16.4, matches that reported in the literature. ${ }^{1}$

\section{(2S)-N-((S)-1-phenylethyl)octan-2-amine (2d)}

Reaction details: $\mathrm{Yb}(\mathrm{OAc})_{3}(10 \mathrm{~mol} \%)$, 2-octanone (0.39 mL, $2.5 \mathrm{mmol}, 1.0$ equiv), $(S)$ $\alpha$-methylbenzylamine $(0.35 \mathrm{~mL}, 2.75 \mathrm{mmol}, 1.1$ equiv $)$, pre-stirred $30 \mathrm{~min}$ at room temperature; hydrogen pressure 120 psi ( 8.3 bar); hydrogenation performed at room temperature. Reaction time: $12 \mathrm{~h} ; 72 \%$ de. Purification by silica gel chromatography $\left(\right.$ Hexane/EtOAc/ $\left./ \mathrm{NH}_{4} \mathrm{OH}=58: 40: 2\right)$ gave the mixture of diastereomers as viscous colorless oil, which then treated with etheral $\mathrm{HCl}$ to obtain the hydrochloride salt $(0.67 \mathrm{~g}$, 83 yield \%). GC (program A, see Experimental section (general remarks) retention time [min]: major $(S, S)-2 \mathbf{d}$ isomer, 10.9; minor $(R, S)-\mathbf{2 d}$ isomer, 10.8 match those reported in the literature. ${ }^{1}$

\section{(2S)-N-((S)1-phenylethyl)hexan-2-amine (2e)}

Reaction details: $\mathrm{Yb}(\mathrm{OAc})_{3}(10 \mathrm{~mol} \%)$, 2-hexanone $(0.31 \mathrm{~mL}, 2.5 \mathrm{mmol}, 1.0$ equiv), (S)$\alpha$-methylbenzylamine $(0.35 \mathrm{~mL}, 2.75 \mathrm{mmol}, 1.1$ equiv $)$, pre-stirred $30 \mathrm{~min}$ at room temperature; hydrogen pressure 8.3 bar (120 psi); hydrogenation performed at room temperature. Reaction time: $12 \mathrm{~h} ; 71 \%$ de. Purification by silica gel flash chromatography $\left(\right.$ Hexane/EtOAc/ $\left.\mathrm{NH}_{4} \mathrm{OH}=89.5: 5.5: 5\right)$ gave the mixture of diastereomers as viscous colorless oil, which then treated with etheral $\mathrm{HCl}$ to obtain the hydrochloride salt $(0.61 \mathrm{~g}$, 82 yield \%). GC (program A, see Experimental section (general remarks)) retention time [min]: major $(S, S)-2 \mathbf{e}$ isomer, 9.7; minor $(R, S)$-2e isomer, 9.6, match those reported in the literature. $^{2}$

\section{(2S)-N-((S)-1-phenylethyl)butan-2-amine (2f)}


Reaction details: $\mathrm{Yb}(\mathrm{OAc})_{3}(10 \mathrm{~mol} \%)$, 2-butanone $(0.22 \mathrm{~mL}, 2.5 \mathrm{mmol}, 1.0$ equiv), $(S)$ a-methylbenzylamine $(0.35 \mathrm{~mL}, 2.75 \mathrm{mmol}, 1.1$ equiv), pre-stirred $30 \mathrm{~min}$ at room temperature; hydrogen pressure 8.3 bar (120 psi); hydrogenation done at room temperature. Reaction time: $12 \mathrm{~h} ; 79 \%$ de. Purification by silica gel chromatography $\left(\right.$ Hexane/EtOAc/ $\left.\mathrm{NH}_{4} \mathrm{OH}=91: 4: 5\right)$ gave the mixture of diastereomers as viscous colorless oil, which then treated with etheral $\mathrm{HCl}$ to obtain the hydrochloride salt $(0.54 \mathrm{~g}, 82$ yield \%). GC (program A, see Experimental section (general remarks)) retention time [min]: major $(S, S)$-2f isomer, 8.5; minor $(R, S)$-2f isomer, 8.4, matches that reported in the literature. $^{2}$

\section{(1S)-N((S)-1-cyclohexylethyl)-1-phenylethanamine (2g)}

Reaction details: $\mathrm{Yb}(\mathrm{OAc})_{3}(10 \mathrm{~mol} \%)$, cyclohexyl methyl ketone $(0.34 \mathrm{~mL}, 2.5 \mathrm{mmol}$, 1.0 equiv), (S)- $\alpha$-MBA $\left(0.35 \mathrm{~mL}, 2.75 \mathrm{mmol}, 1.1\right.$ equiv), pre-stirred $30 \mathrm{~min}$ at $50{ }^{\circ} \mathrm{C}$; hydrogen pressure 20 bar (290 psi); hydrogenation performed at $50{ }^{\circ} \mathrm{C}$. Reaction time: 12 h; $98 \%$ de. Purification by silica gel flash chromatography (Hexane/EtOAc/ $\mathrm{NH}_{4} \mathrm{OH}=$ $83: 15: 2)$ gave the mixture of diastereomers as a viscous colorless oil, which when treated with etheral $\mathrm{HCl}$ provided the hydrochloride salt $(0.60 \mathrm{~g}, 81$ yield \%). GC (program D, see Experimental section (general remarks)) retention time [min]: major $(S, S)-\mathbf{2 g}$ isomer, 14.9; minor $(R, S)-2 \mathrm{~g}$ isomer, 14.7 , matches that reported in the literature. ${ }^{1}$

\section{(2S)-3-Methyl-N- ((S)-1-phenylethyl) butan-2-amine (2h)}

Reaction details: $\mathrm{Yb}(\mathrm{OAc})_{3}(10 \mathrm{~mol} \%), 3$-methyl-2-butanone $(0.27 \mathrm{~mL}, 2.5 \mathrm{mmol}, 1.0$ equiv), (S)- $\alpha$-methylbenzylamine $(0.35 \mathrm{~mL}, 2.75 \mathrm{mmol}, 1.1$ equiv), pre-stirred $30 \mathrm{~min}$ at $50{ }^{\circ} \mathrm{C}$; hydrogen pressure 20 bar $(290 \mathrm{psi})$; hydrogenation performed at $50{ }^{\circ} \mathrm{C}$. Reaction time: $12 \mathrm{~h} ; 98 \%$ de. Purification by silica gel flash chromatography $\left(\mathrm{Hexane} / \mathrm{EtOAc} / \mathrm{NH}_{4} \mathrm{OH}=92.5: 3.5: 4\right)$ gave the mixture of diastereomers as a viscous 
colorless oil, which then treated with etheral $\mathrm{HCl}$ provided the hydrochloride salt $(0.54 \mathrm{~g}$,

78 yield \%). GC (program D, see Experimental section (general remarks)) retention time [min]: major $(S, S)-2 \mathbf{h}$ isomer, 11.2; minor $(R, S)-2 \mathbf{h}$ isomer, 11.1, matches that reported in the literature. ${ }^{1}$

\section{Bis((S)-1-phenylethyl)amine (2i)}

Reaction details: $\mathrm{Yb}(\mathrm{OAc})_{3}(10 \mathrm{~mol} \%)$, acetophenone $(0.29 \mathrm{~mL}, 2.5 \mathrm{mmol}, 1.0$ equiv), (S)- $\alpha$-methylbenzylamine $\left(0.35 \mathrm{~mL}, 2.75 \mathrm{mmol}, 1.1\right.$ equiv), pre-stirred $30 \mathrm{~min}$ at $50{ }^{\circ} \mathrm{C}$; hydrogen pressure 20 bar (290 psi); hydrogenation performed at $50{ }^{\circ} \mathrm{C}$. Reaction time: 12 h; $94 \%$ de. Purification by silica gel flash chromatography (Hexane/EtOAc/ $\mathrm{NH}_{4} \mathrm{OH}=$ 74:25:1) gave the mixture of diastereomers as a viscous colorless oil, which then treated with etheral $\mathrm{HCl}$ to obtain the hydrochloride salt $(0.41 \mathrm{~g}, 63$ yield \%). GC (program D, see Experimental section (general remarks)) retention time [min]: major $(S, S)-2 \mathbf{i}$ isomer, 14.4; minor $(R, S)-2 \mathbf{i}$ isomer, 14.7 , matches that reported in the literature. ${ }^{2}$

\section{(2S)-3,3-dimethyl-N-((S)-1-phenylethyl)butan-2-amine (2b)}

Reaction details: $\mathrm{Yb}(\mathrm{OAc})_{3}(10 \mathrm{~mol} \%), 3,3$-dimethyl-2-butanone $(0.31 \mathrm{~mL}, 2.5 \mathrm{mmol}$, 1.0 equiv), (S)- $\alpha$-methylbenzylamine $(0.35 \mathrm{~mL}, 2.75 \mathrm{mmol}, 1.1$ equiv), pre-stirred $4 \mathrm{~h}$ at $50{ }^{\circ} \mathrm{C}$; then adding $\mathrm{Pt} / \mathrm{C}$ (instead of Raney $\mathrm{Ni}$ ) in four equal portions at $\mathrm{t}=0,6,12,20 \mathrm{~h}$ (total added Pt equals $1.0 \mathrm{~mol} \%$ ), with a total hydrogenation time of $30 \mathrm{~h}$ at $8.3 \mathrm{bar}(120$ psi) and at $50{ }^{\circ} \mathrm{C} .92 \%$ de. Purification by silica gel flash chromatography $\left(\right.$ Hexane/EtOAc/ $\left.\mathrm{NH}_{4} \mathrm{OH}=94.5: 1.5: 4\right)$ gave the mixture of diastereomers as a viscous colorless oil, which when treated with etheral $\mathrm{HCl}$ to obtain the hydrochloride salt $(0.58$ g, 78 yield \%). GC (program C, see Experimental section (general remark)) retention time [min]: major $(S, S)-\mathbf{2 b}$ isomer, 11.8; minor $(R, S)-2 \mathbf{b}$ isomer, 11.6, matches that reported in the literature. ${ }^{2}$ 


\section{General Procedure: Brønsted acids (normal de)}

The reaction vessel was evacuated under high vacuum for $5 \mathrm{~min}$ before flooding with nitrogen. To the vessel, anhydrous methanol (2.5 mL, $1.0 \mathrm{M})$, acetic acid (20 mol \%), ketone (2.5 mmol, 1.0 equiv) (1), and $(S)$ - $\alpha$-methylbenzylamine $(0.35 \mathrm{~mL}, 2.75 \mathrm{mmol}$, 1.1 equiv) were added and stirred for $20-30 \mathrm{~min}$ at the temperature at which the hydrogenation was performed at $\left(22\right.$ or $\left.50{ }^{\circ} \mathrm{C}\right)$. The remaining procedural details should be followed as in the section entitled: "General procedure: Catalytic $\mathrm{Yb}(\mathrm{OAc})_{3}$, $\mathrm{Y}(\mathrm{OAc})_{3}$, or $\mathrm{Ce}(\mathrm{OAc})_{3}$ (normal de)."

\section{(2S)-4-methyl-N-((S)-1-phenylethyl)pentan-2-amine (2a)}

Reaction details: 4-Methyl-2-pentanone (0.31 mL, $2.5 \mathrm{mmol}, 1.0$ equiv), (S)- $\alpha$ methylbenzylamine $(0.35 \mathrm{~mL}, 2.75 \mathrm{mmol}, 1.1$ equiv), acetic acid $(0.028 \mathrm{~mL}, 20 \mathrm{~mol} \%)$, pre-stirred $30 \mathrm{~min}$ at $50{ }^{\circ} \mathrm{C}$; hydrogen pressure 8.3 bar (120 psi); hydrogenation performed at $50{ }^{\circ} \mathrm{C}$. Reaction time: $12 \mathrm{~h} ; 92 \%$ de. Purification by silica gel flash chromatography (Hexane/EtOAc/ $\left.\mathrm{NH}_{4} \mathrm{OH}=87: 9: 4\right)$ gave the mixture of diastereomers as a viscous colorless oil, which when treated with etheral $\mathrm{HCl}$ provided the hydrochloride salt (0.60 g, 80 yield \%). GC (program D, see Experimental section (general remarks)) retention time [min]: major $(S, S)-2 \mathbf{a}$ isomer, 11.8; minor $(R, S)$-2a isomer, 11.6, matches that reported in the literature. ${ }^{1}$

\section{(2S)-N-((S)-1-phenylethyl)octan-2-amine (2d)}

Reaction details: 2-Octanone (0.39 mL, $2.5 \mathrm{mmol}, 1.0$ equiv), $(S)$ - $\alpha$-methylbenzylamine (0.35 mL, $2.75 \mathrm{mmol}, 1.1$ equiv), acetic acid (0.028 mL, $20 \mathrm{~mol} \%)$, pre-stirred $30 \mathrm{~min}$ at room temperature; hydrogen pressure 8.3 bar (120 psi); hydrogenation performed at room temperature. Reaction time: $12 \mathrm{~h} ; 72 \%$ de. Purification by silica gel chromatography 
$\left(\mathrm{Hexane} / \mathrm{EtOAc} / \mathrm{NH}_{4} \mathrm{OH}=58: 40: 2\right)$ gave the mixture of diastereomers as viscous colorless oil, which when treated with etheral $\mathrm{HCl}$ provided the hydrochloride salt $(0.68$ g, 83 yield \%). GC (program A, see Experimental sectuib (general remarks)) retention time [min]: major $(S, S)-\mathbf{2 d}$ isomer, 10.9; minor $(R, S)-\mathbf{2 d}$ isomer, 10.8, matches that reported in the literature. ${ }^{1}$

\section{(1S)-N((S)-1-cyclohexylethyl)-1-phenylethanamine (2g):}

Reaction details: Cyclohexyl methyl ketone (0.34 mL, $2.5 \mathrm{mmol}, 1.0$ equiv), (S)- $\alpha$-MBA (0.35 mL, $2.75 \mathrm{mmol}, 1.1$ equiv), acetic acid $(0.028 \mathrm{~mL}, 20 \mathrm{~mol} \%)$, pre-stirred $30 \mathrm{~min}$ at $50{ }^{\circ} \mathrm{C}$; hydrogen pressure 20 bar (290 psi); hydrogenation done at $50{ }^{\circ} \mathrm{C}$. Reaction time: $12 \mathrm{~h} ; 98 \%$ de. Purification by silica gel flash chromatography (Hexane/EtOAc/ $\mathrm{NH}_{4} \mathrm{OH}=$ $83: 15: 2)$ gave the mixture of diastereomers as a viscous colorless oil, which when treated with etheral $\mathrm{HCl}$ provided the hydrochloride salt $(0.62 \mathrm{~g}, 82$ yield \%). GC (program D, see Experimental section (general remarks)) retention time [min]: major $(S, S)-\mathbf{2 g}$ isomer, 14.9; minor $(R, S)-2 \mathrm{~g}$ isomer, 14.7 , matches that reported in the literature. ${ }^{1}$

\section{Bis((S)-1-phenylethyl)amine (2i)}

Reaction details: Acetophenone (0.29 mL, $2.5 \mathrm{mmol}, 1.0$ equiv), (S)- $\alpha-$ methylbenzylamine $(0.35 \mathrm{~mL}, 2.75 \mathrm{mmol}, 1.1$ equiv), acetic acid $(0.028 \mathrm{~mL}, 20 \mathrm{~mol} \%)$, pre-stirred $30 \mathrm{~min}$ at $50{ }^{\circ} \mathrm{C}$; hydrogen pressure $20 \mathrm{bar}$ (290 psi); hydrogenation performed at $50{ }^{\circ} \mathrm{C}$. Reaction time: $12 \mathrm{~h} ; 93 \%$ de. Purification by silica gel flash chromatography $\left(\right.$ Hexane/EtOAc/ $\left.\mathrm{NH}_{4} \mathrm{OH}=74: 25: 1\right)$ gave the mixture of diastereomers as a viscous colorless oil, which when treated with etheral $\mathrm{HCl}$ provided the hydrochloride salt $(0.37$ g, 55 yield \%). GC (program D, see Experimental section (general remarks)) retention time [min]: major $(S, S)-2 \mathbf{i}$ isomer, 14.4 ; minor $(R, S)-2 \mathbf{i}$ isomer, 14.7, matches that reported in the literature. ${ }^{2}$ 


\section{References and Notes}

(1) Nugent, T. C.; Wakchaure, V. N.; Ghosh, A. K.; Mohanty, R. R. Org. Lett. 2005, 7, 4967.

(2) Nugent, T. C.; Ghosh, A. K.; Wakchaure, V. N.; Mohanty, R. R. Adv. Synth. Catal. 2006, 348, 1289.

(3) Davis, B. A.; Durden, D. A. Synth. Commun. 2001, 31, 569.

(4) Gottlieb, H. E.; Kotlyar, V.; Nudelman, A. J. Org. Chem. 1997, 62, 7512.

\section{Spectral data}

All reductive amination products (2) and primary amine 4d have been previously characterized. Gas chromatograms (for de or ee determination) are provided for all compounds. For those reactions showing enhanced de [stoichiometric $\mathrm{Yb}(\mathrm{OAc})_{3}$ ], the ${ }^{1} \mathrm{H}$ and ${ }^{13} \mathrm{C}$ NMR spectra are additionally provided, specifically for $\mathbf{2 a}, \mathbf{2 b}, \mathbf{2 c}, \mathbf{2 d}, \mathbf{2 e}, \mathbf{2 f}$. 


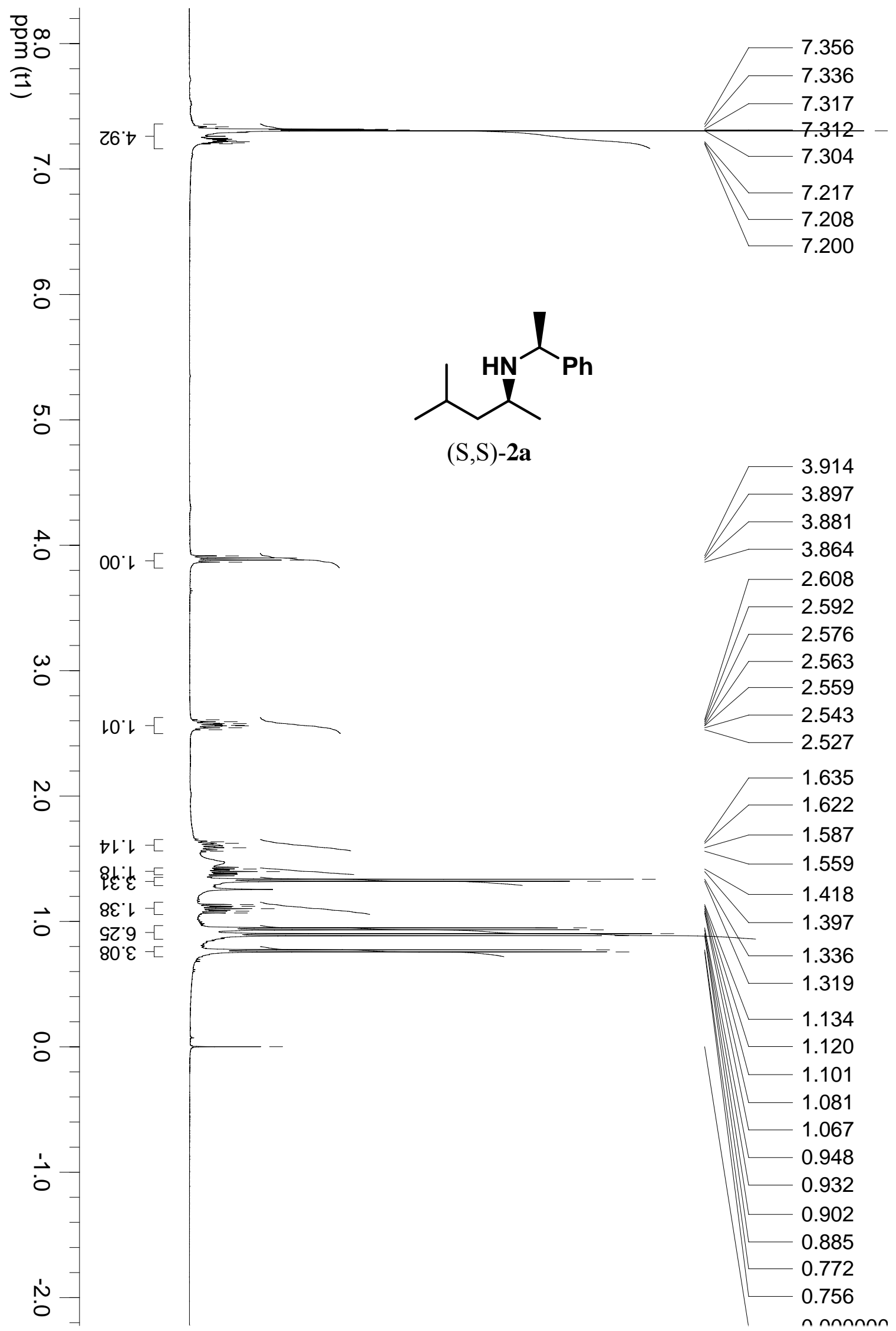









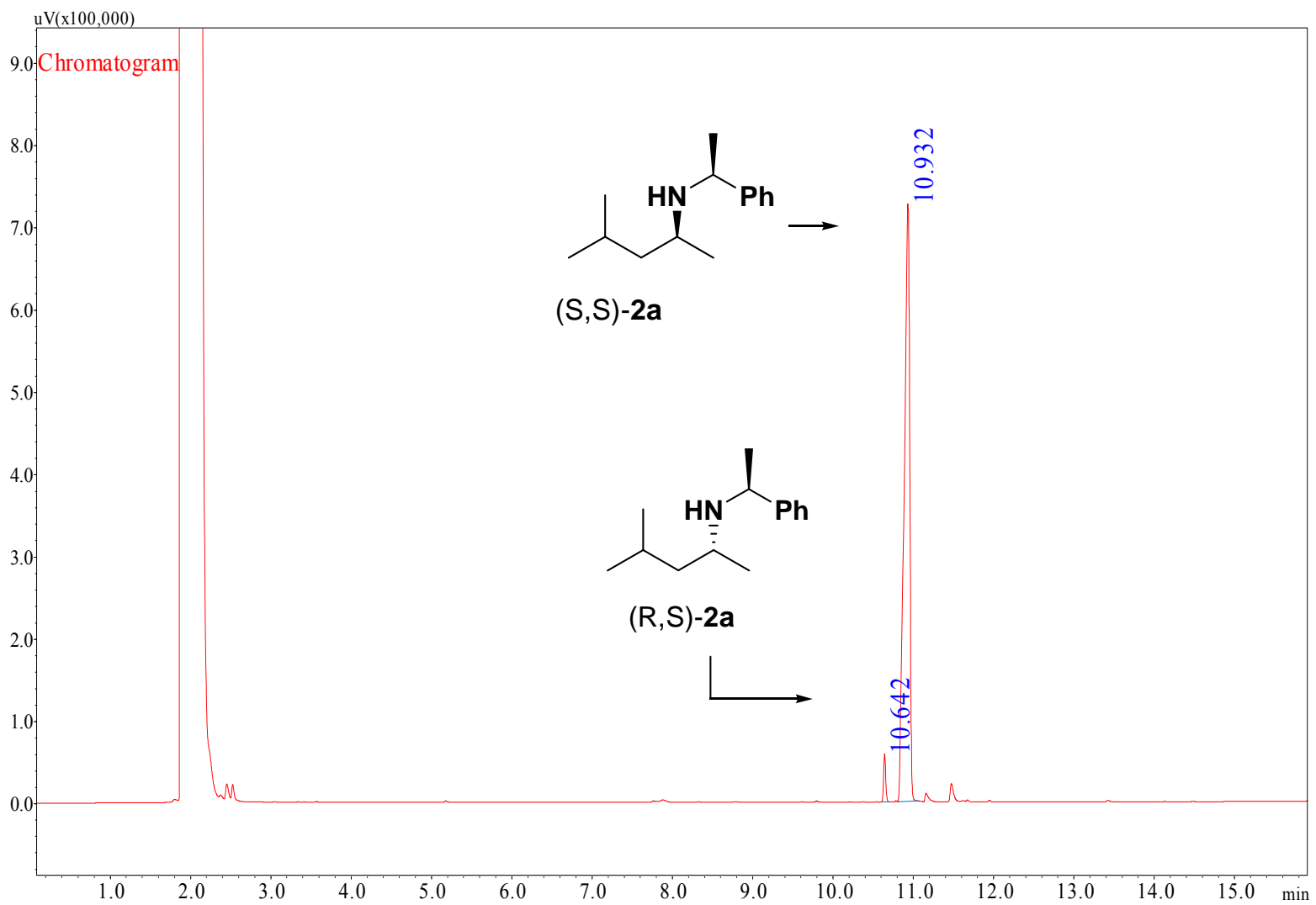

\section{4\% de}

\begin{tabular}{|l|l|l|l|l|}
\hline \multicolumn{5}{|c|}{ Peak Table-Channel 1 } \\
\hline Peak & Ret. Time & Area & Height & Conc. \\
\hline 1 & 10.642 & 103801.0 & 57464.3 & 2.90149 \\
\hline 2 & 10.932 & 3473709.0 & 725649.2 & 97.09851 \\
\hline
\end{tabular}




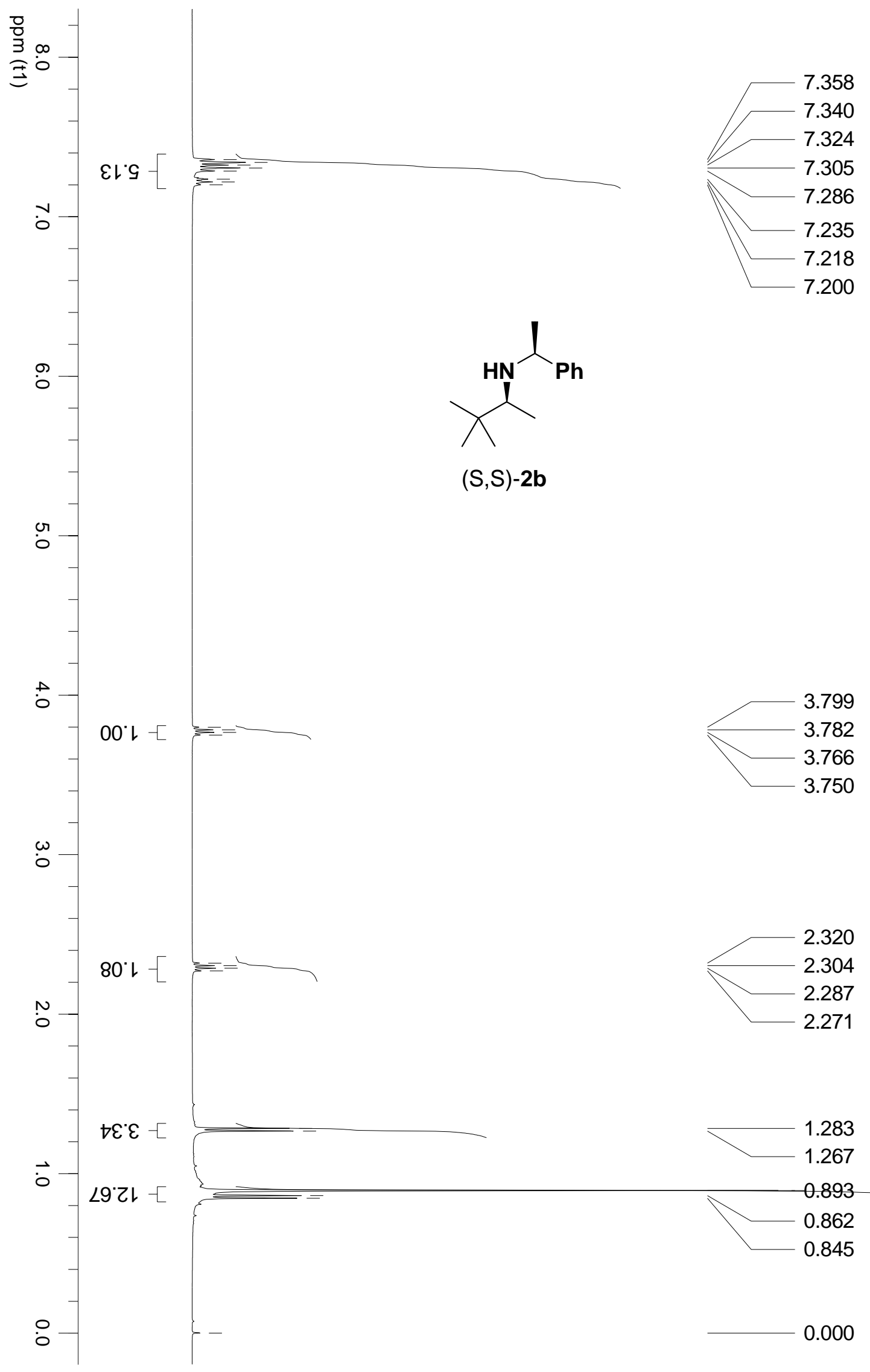




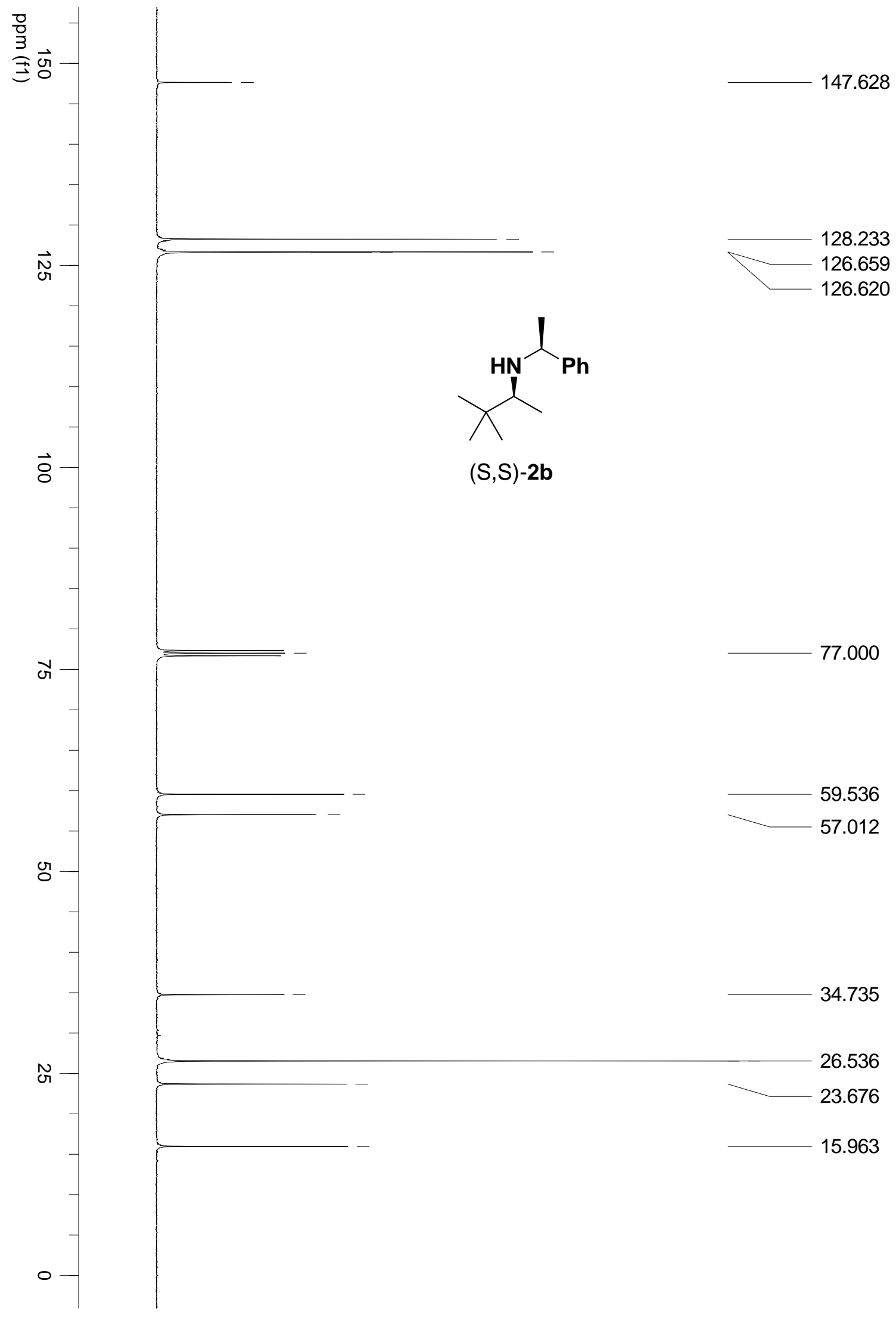




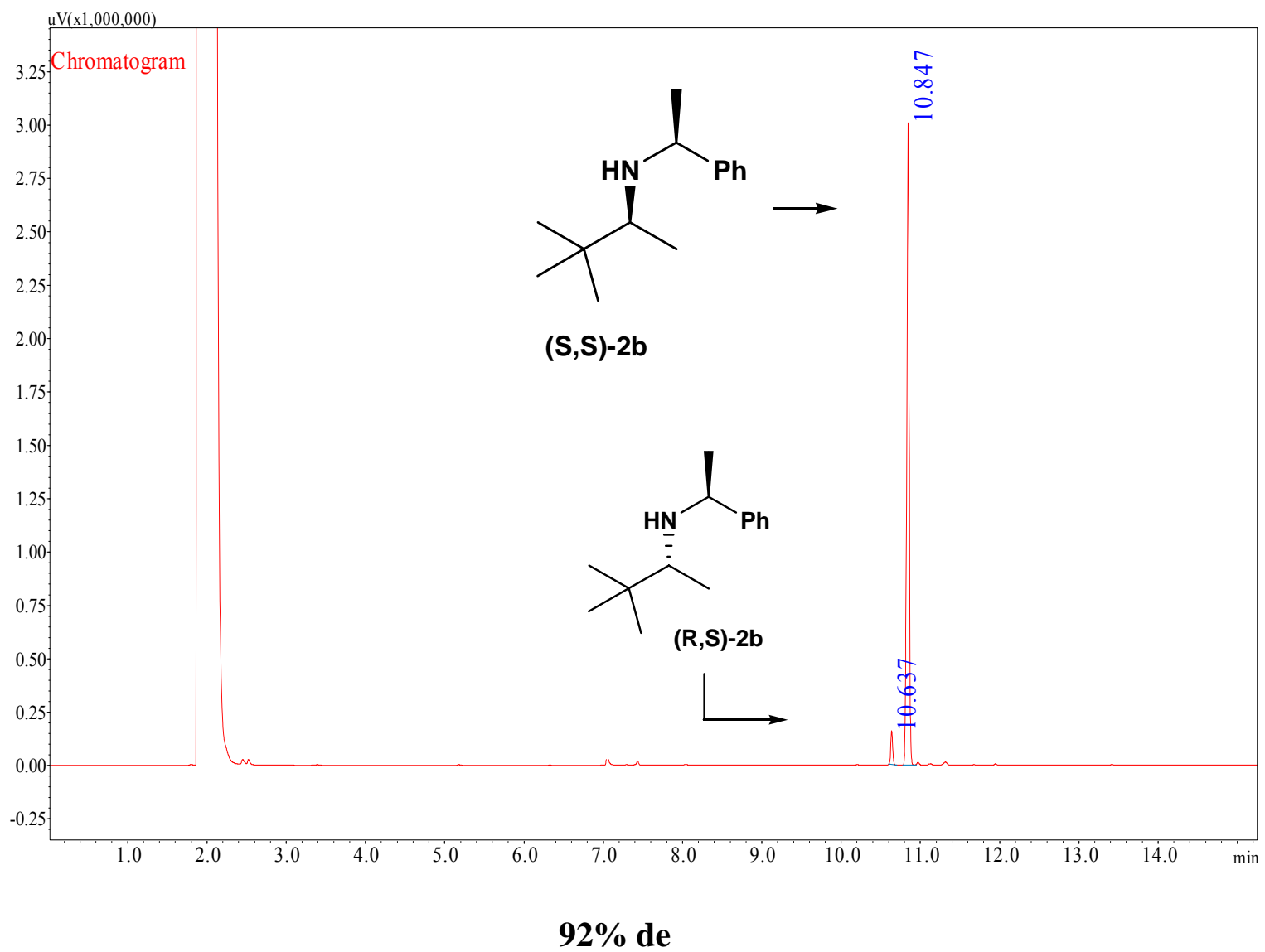

\begin{tabular}{|l|l|l|l|l|}
\hline \multicolumn{5}{|c|}{ Peak Table-Channel 1 } \\
\hline Peak & Ret. Time & Area & Height & Conc. \\
\hline 1 & 10.637 & 268603.4 & 154845.2 & 4.09932 \\
\hline 2 & 10.847 & 6283778.8 & 3002789.0 & 95.90068 \\
\hline
\end{tabular}




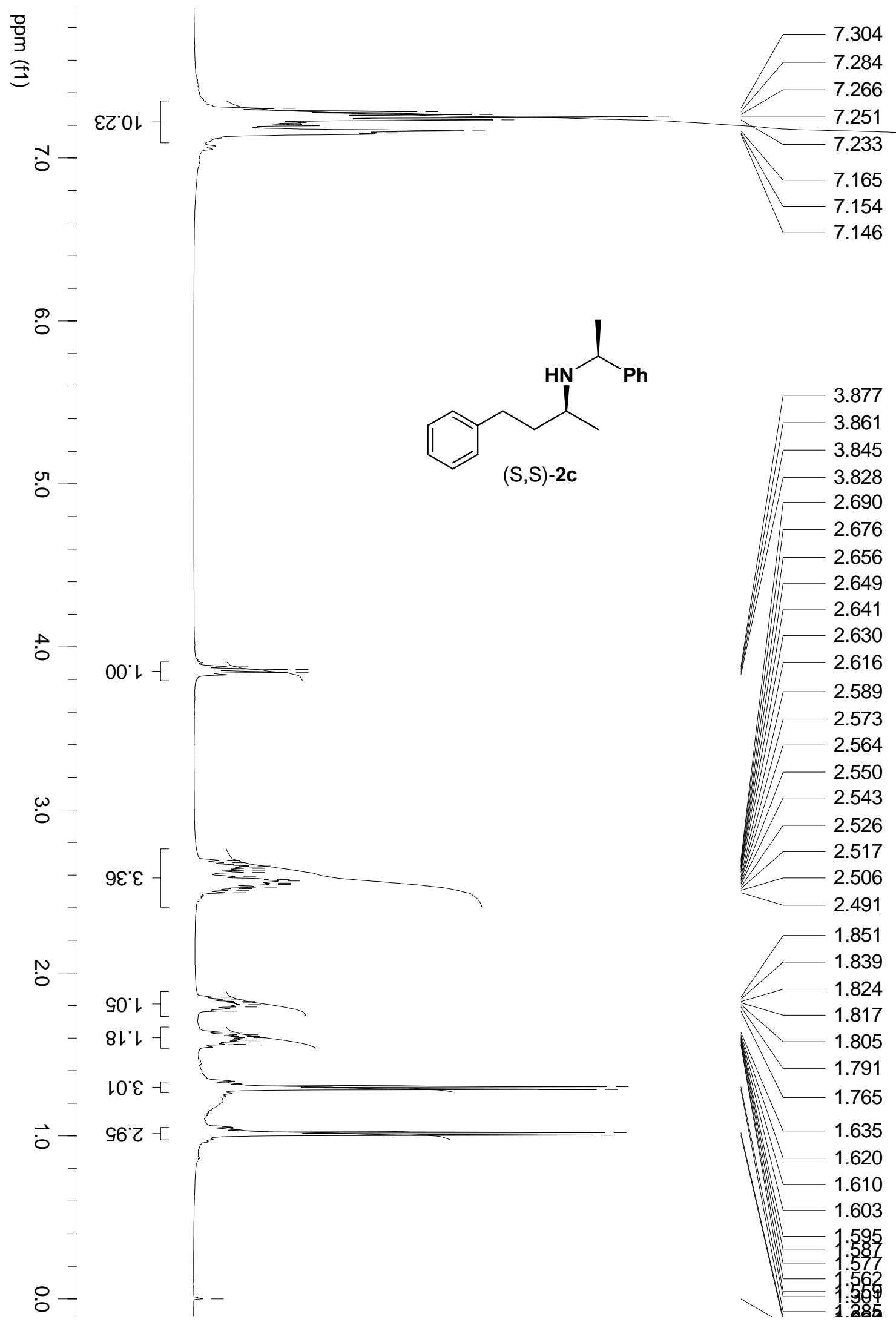




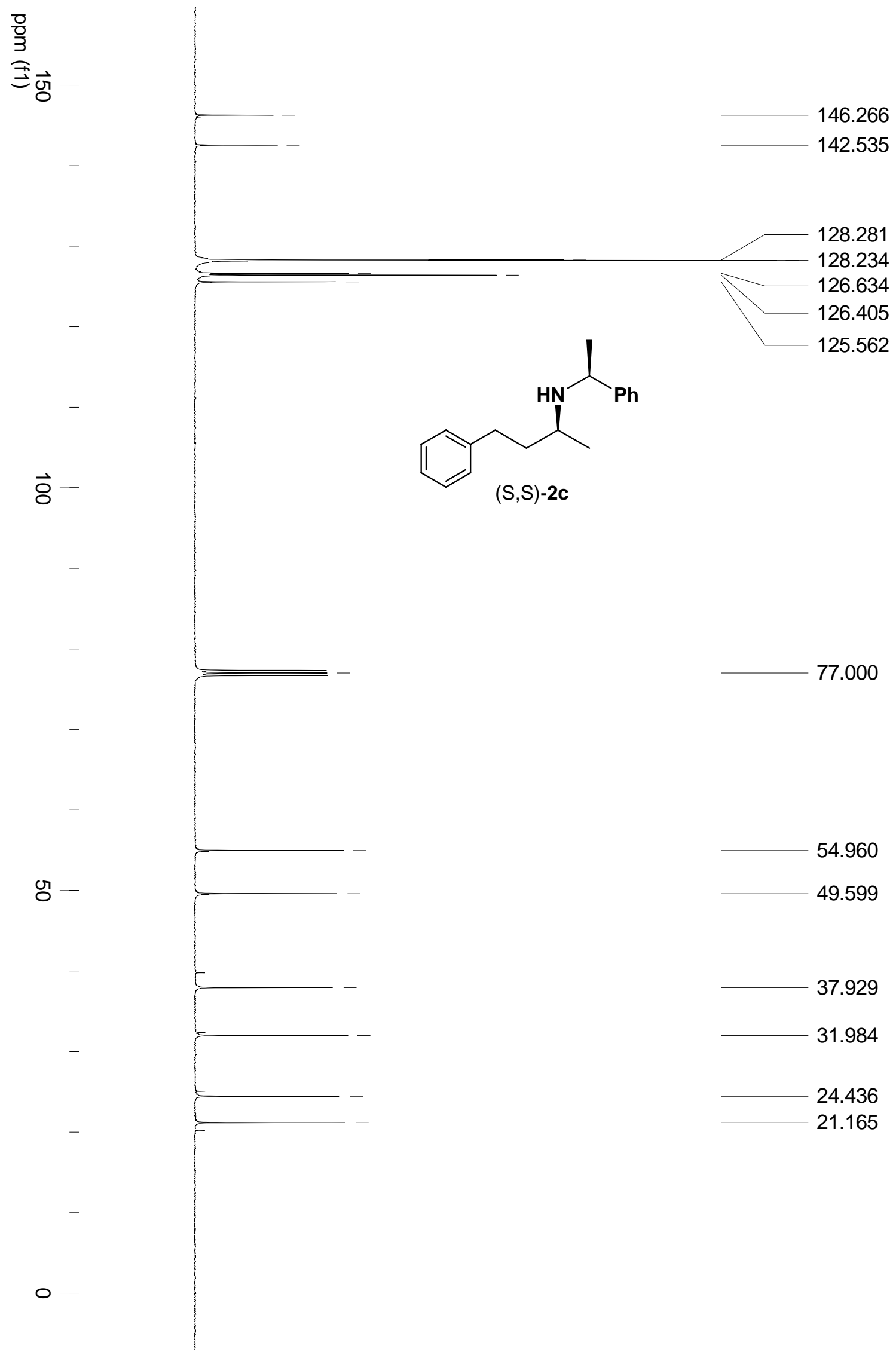




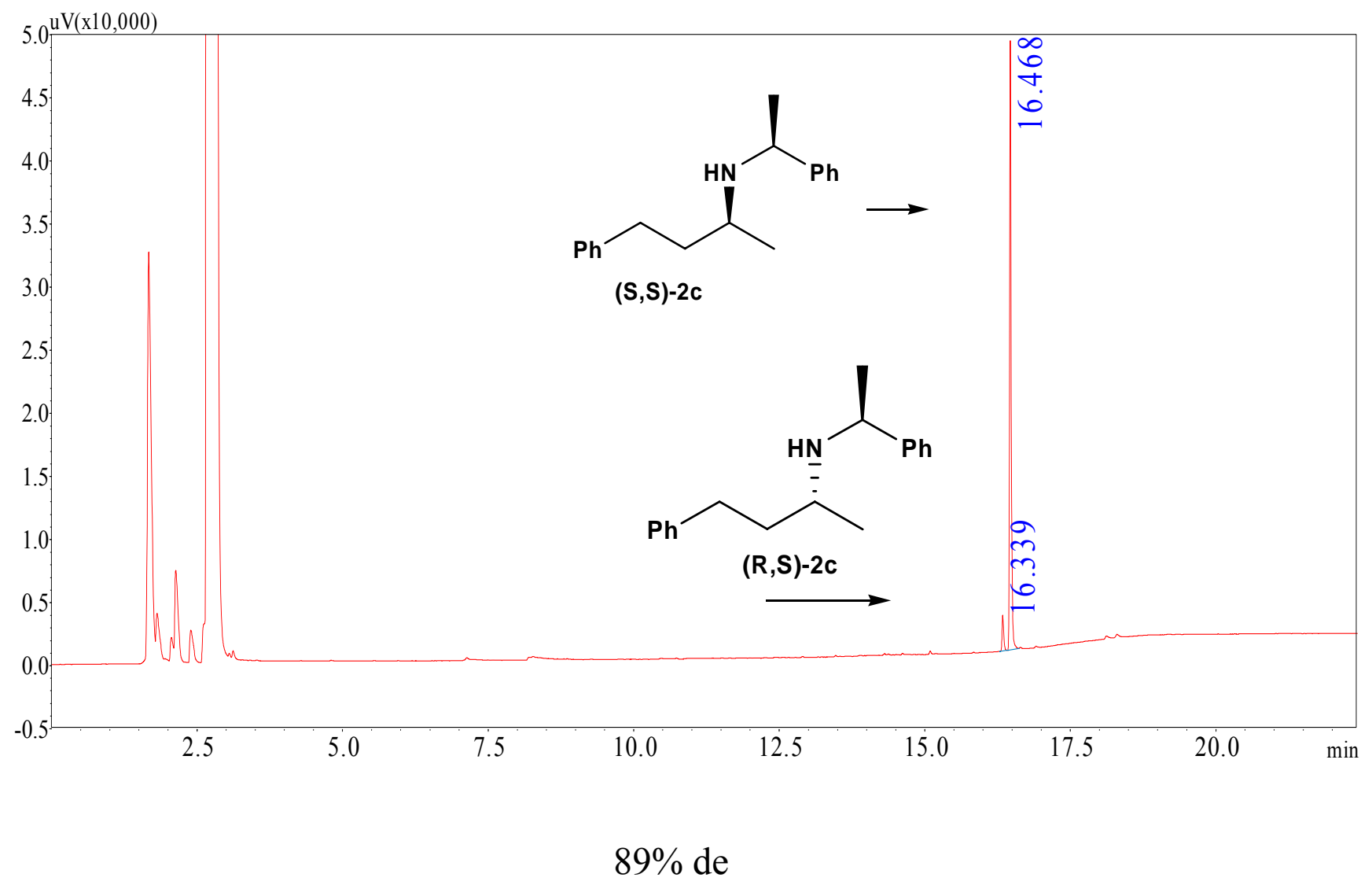

\begin{tabular}{|l|l|l|l|l|}
\hline \multicolumn{5}{|c|}{ Peak Table-Channel 1 } \\
\hline Peak & Ret. Time & Area & Height & Conc. \\
\hline 1 & 16.339 & 5527.4 & 5.61245 & 5.6125 \\
\hline 2 & 16.468 & 92956.8 & 94.38755 & 94.3875 \\
\hline
\end{tabular}




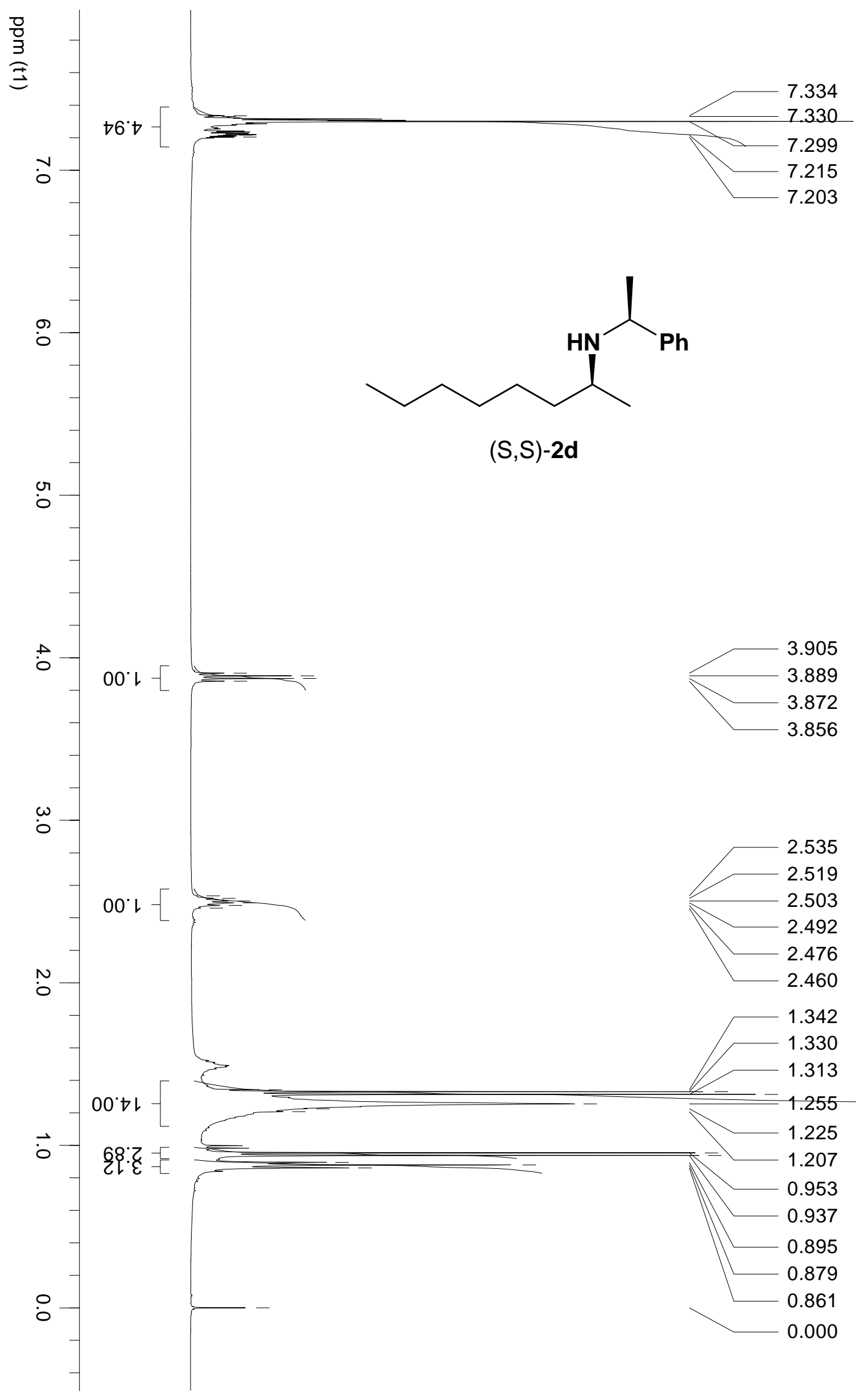




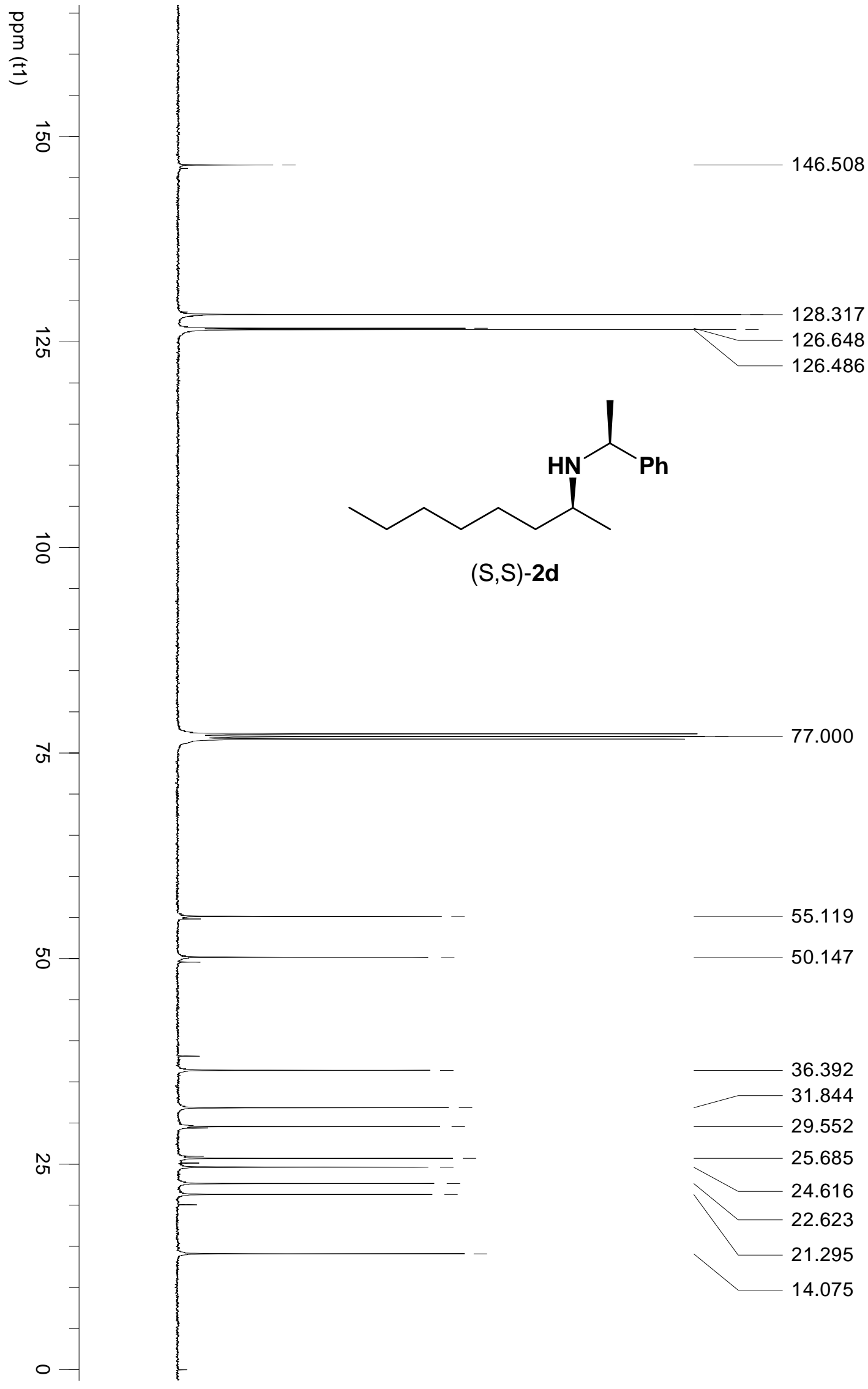




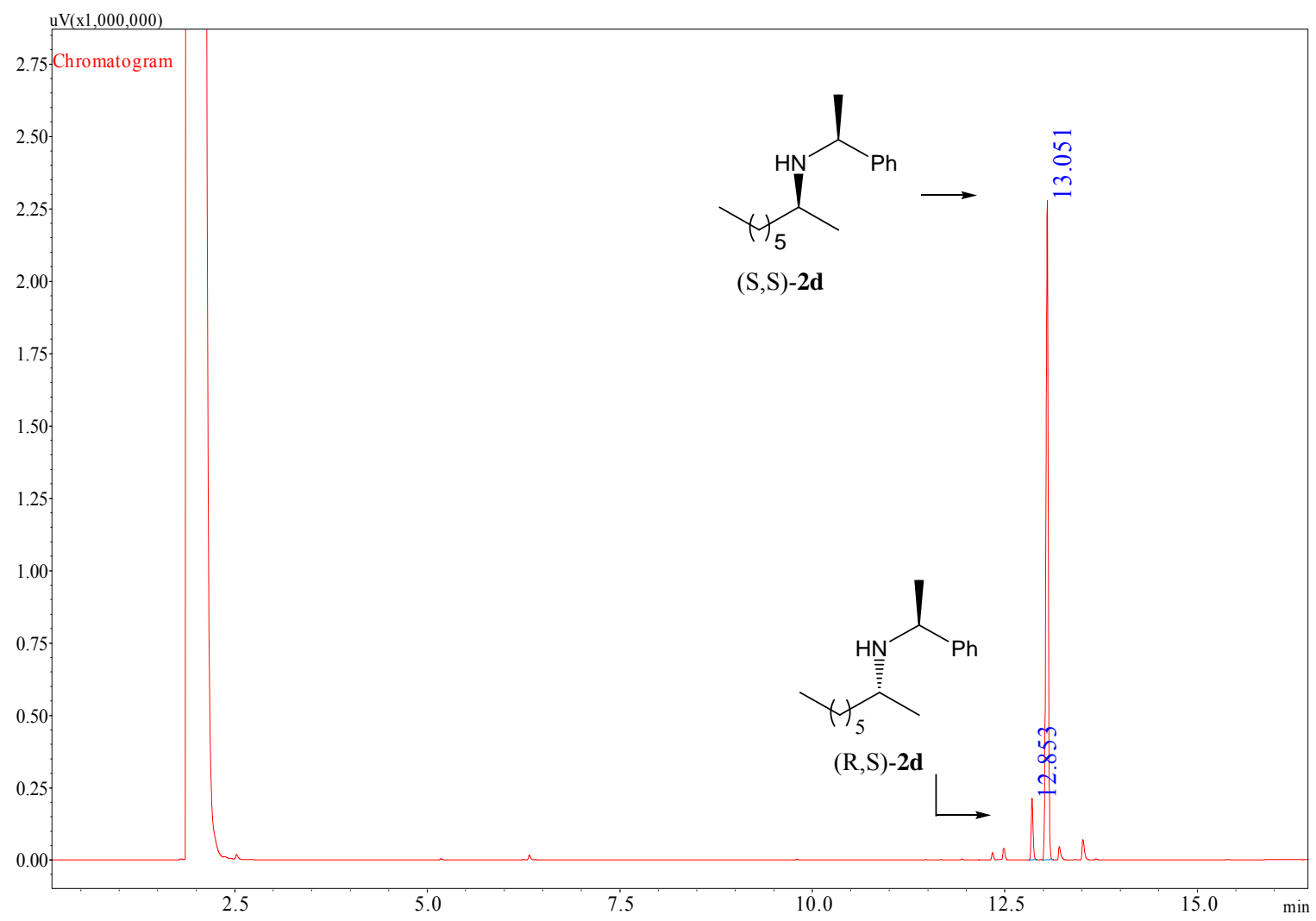

$87 \%$ de

\begin{tabular}{|l|l|l|l|l|}
\hline \multicolumn{5}{|c|}{ Peak Table-Channel 1 } \\
\hline Peak & Ret. Time & Area & Height & Conc. \\
\hline 1 & 12.853 & 76004.0 & 50073.5 & 6.56171 \\
\hline 2 & 13.051 & 1082290.8 & 703688.0 & 93.43829 \\
\hline
\end{tabular}




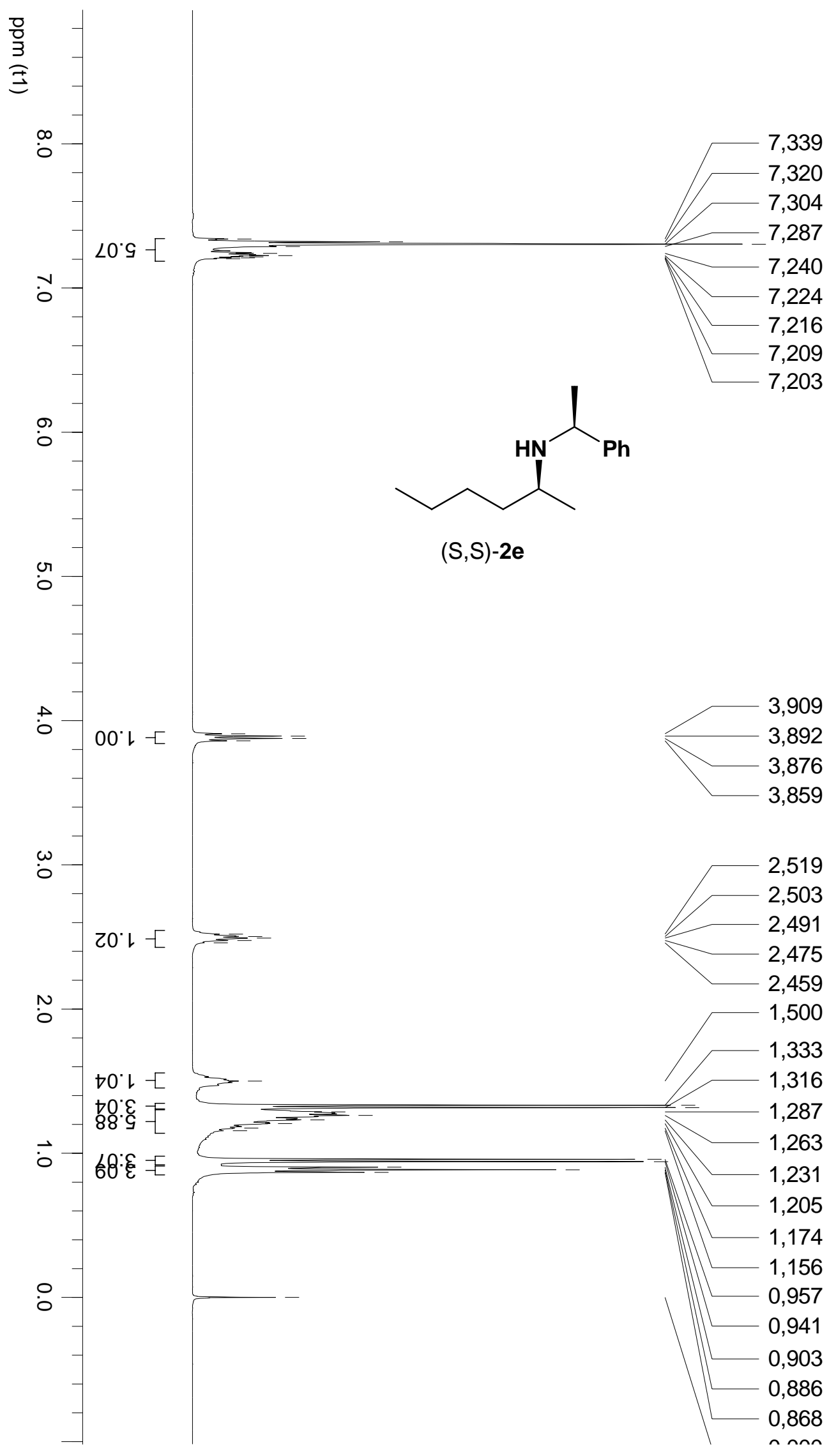




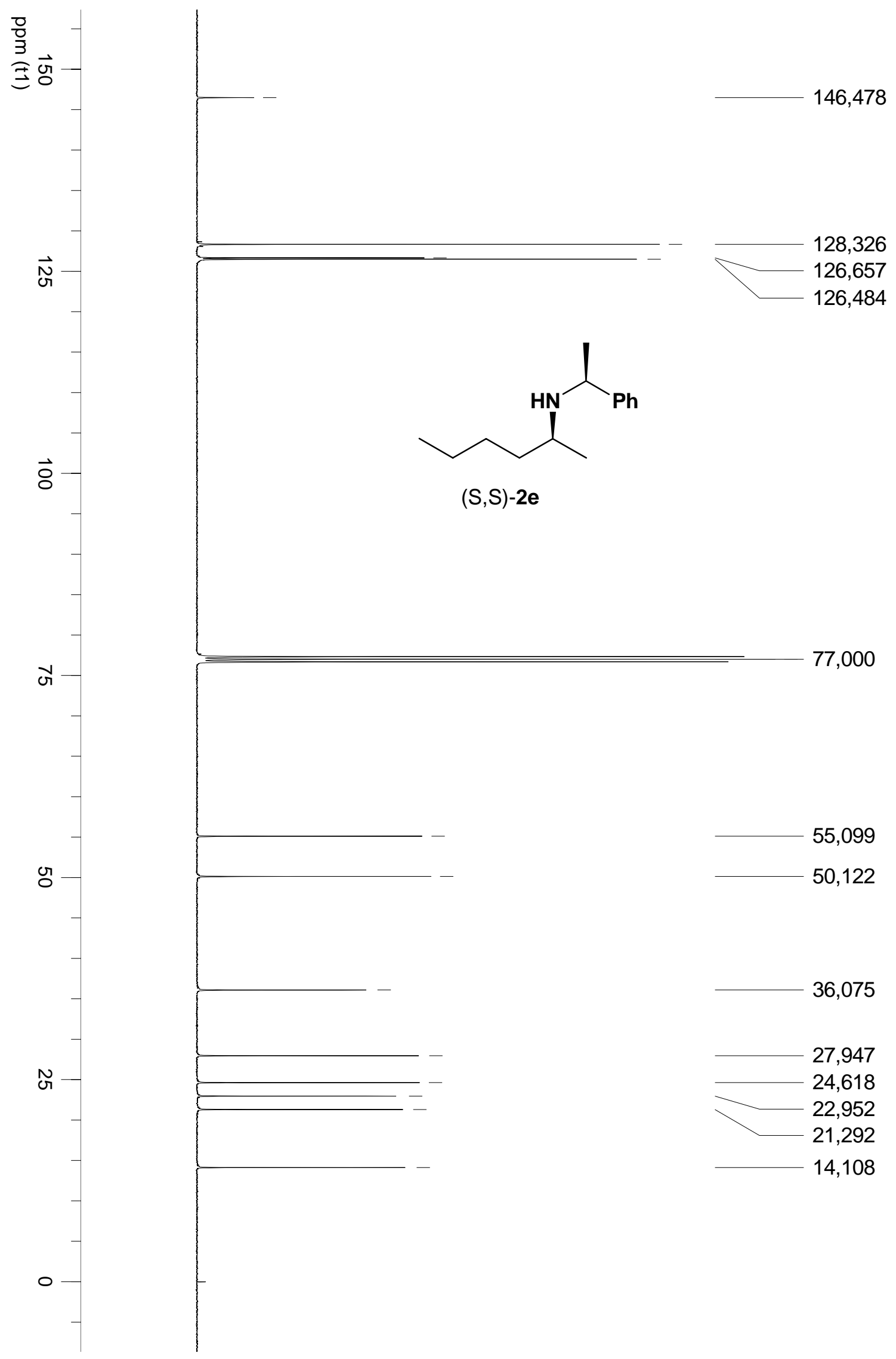




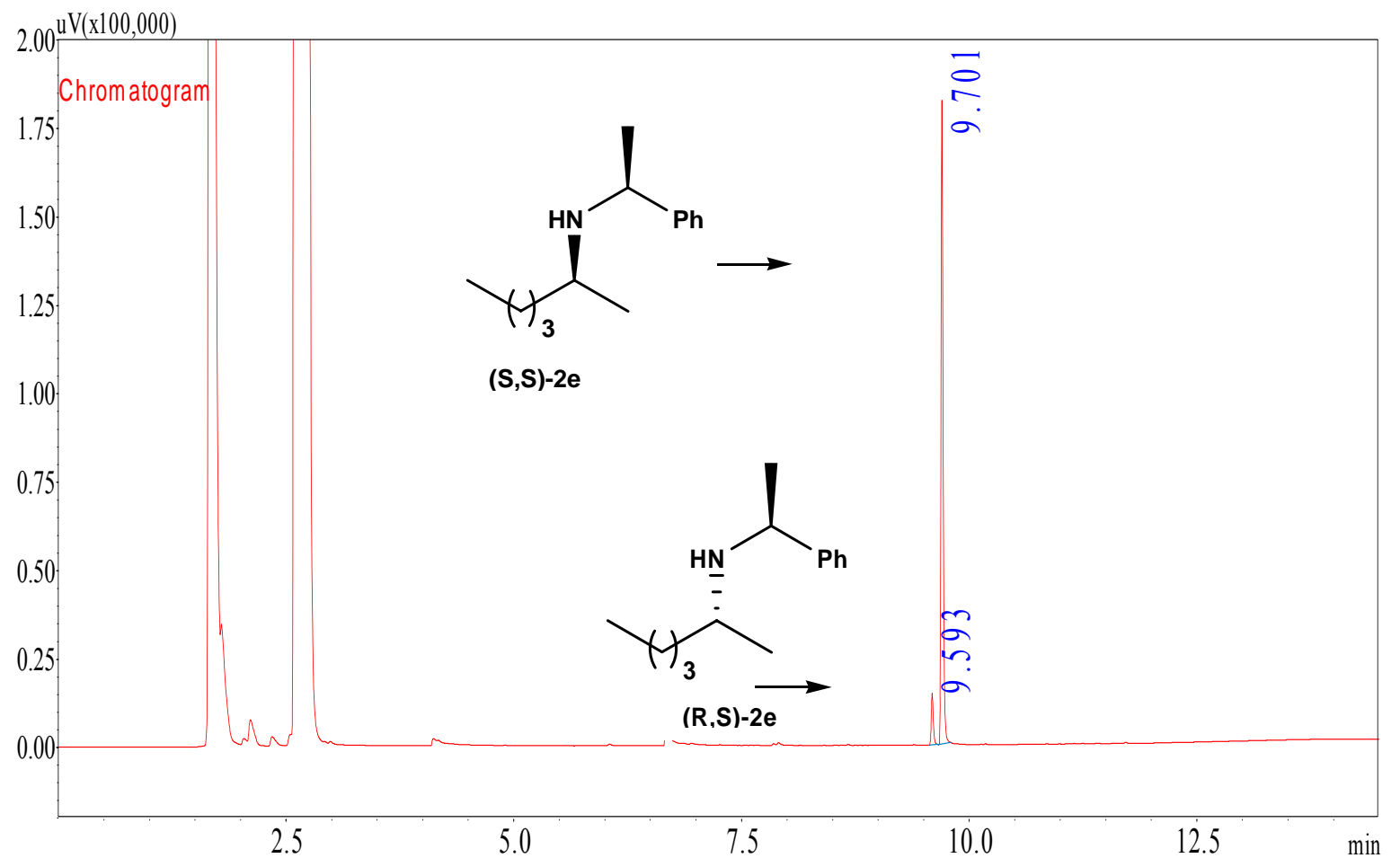

$85 \%$ de

\begin{tabular}{|l|l|l|l|l|}
\hline \multicolumn{5}{|c|}{ Peak Table-Channel 1 } \\
\hline Peak & Ret. Time & Area & Height & Conc. \\
\hline 1 & 9.593 & 22445.5 & 14498.9 & 7.35078 \\
\hline 2 & 9.701 & 282902.7 & 181680.0 & 92.64922 \\
\hline
\end{tabular}




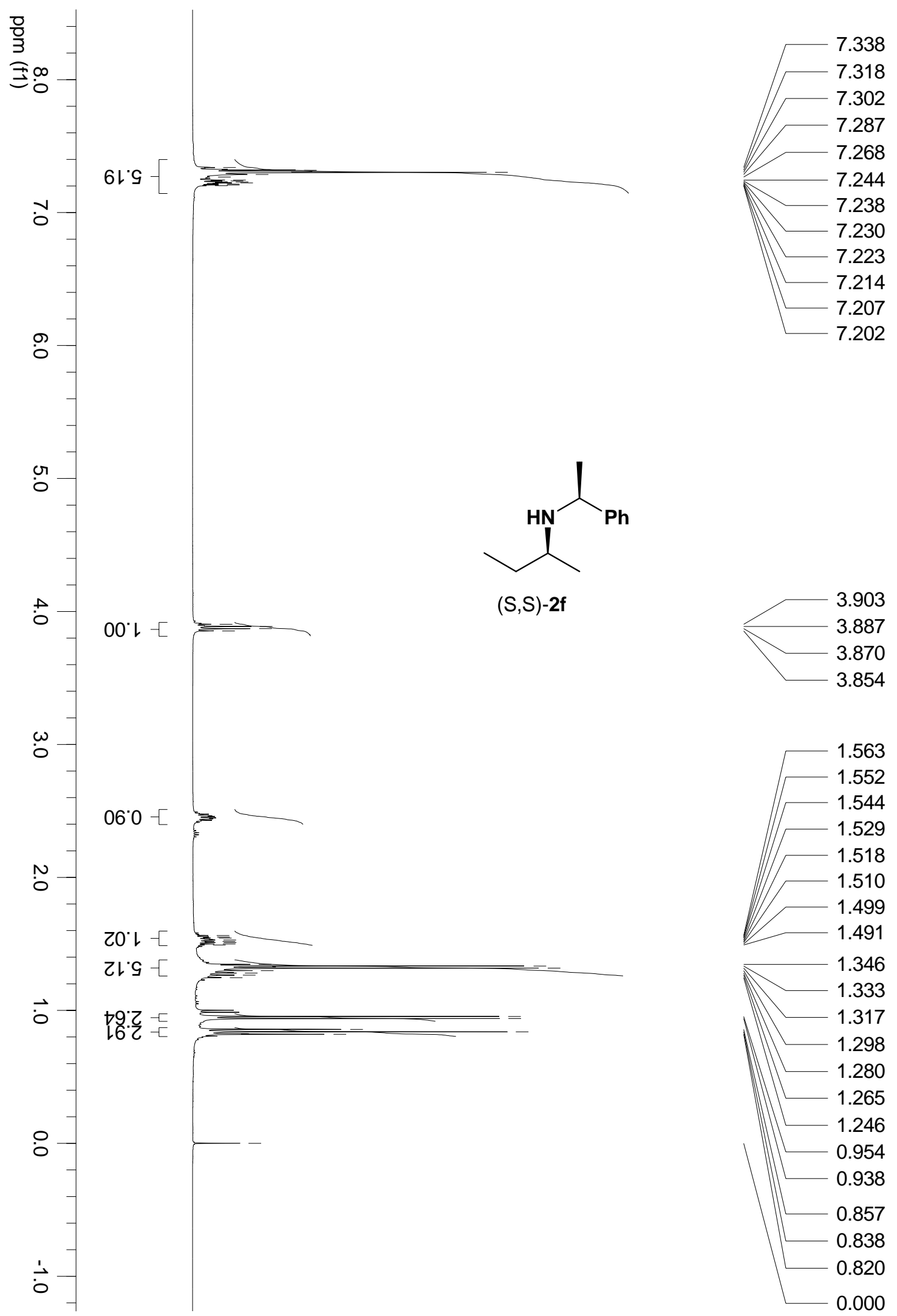




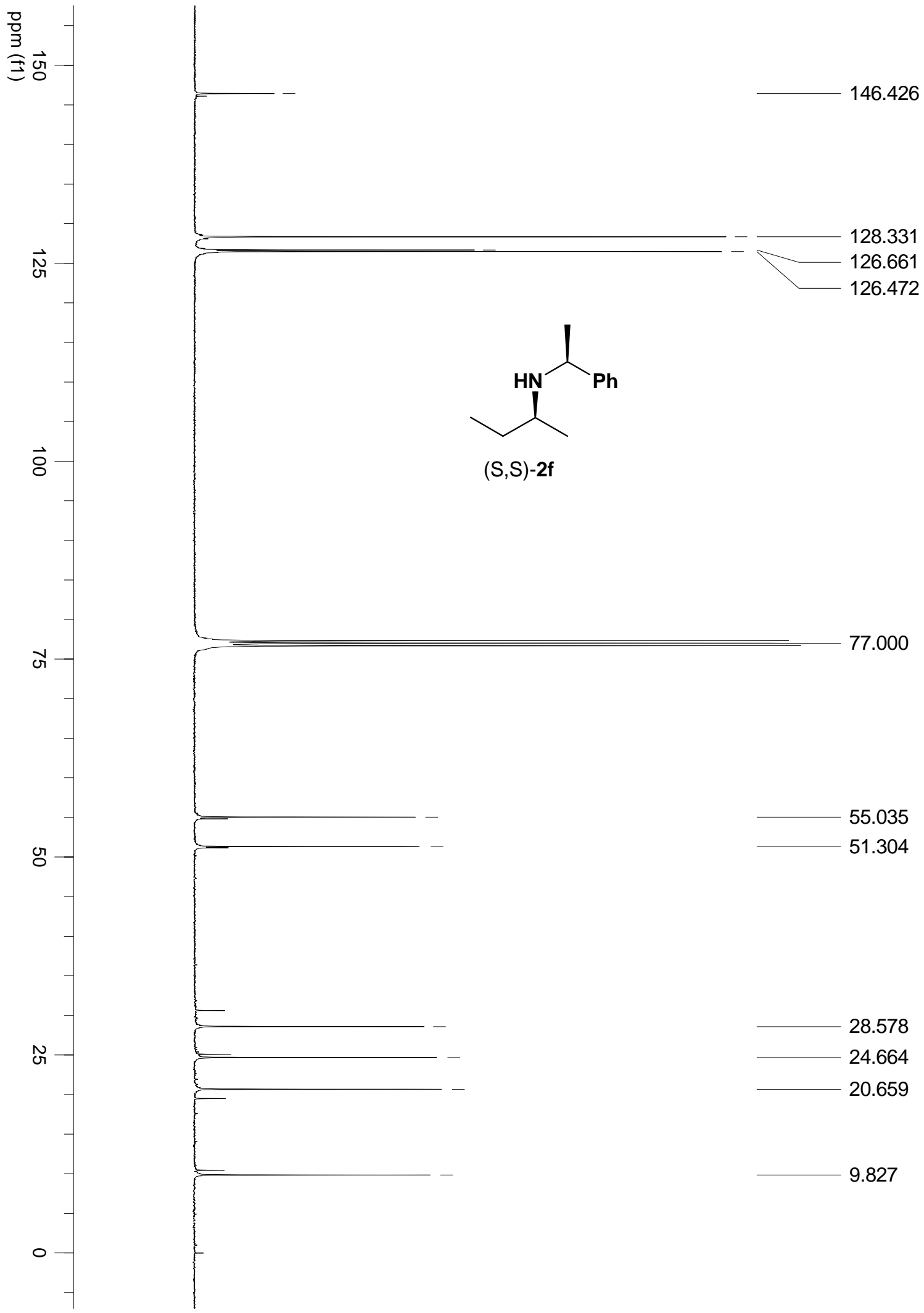




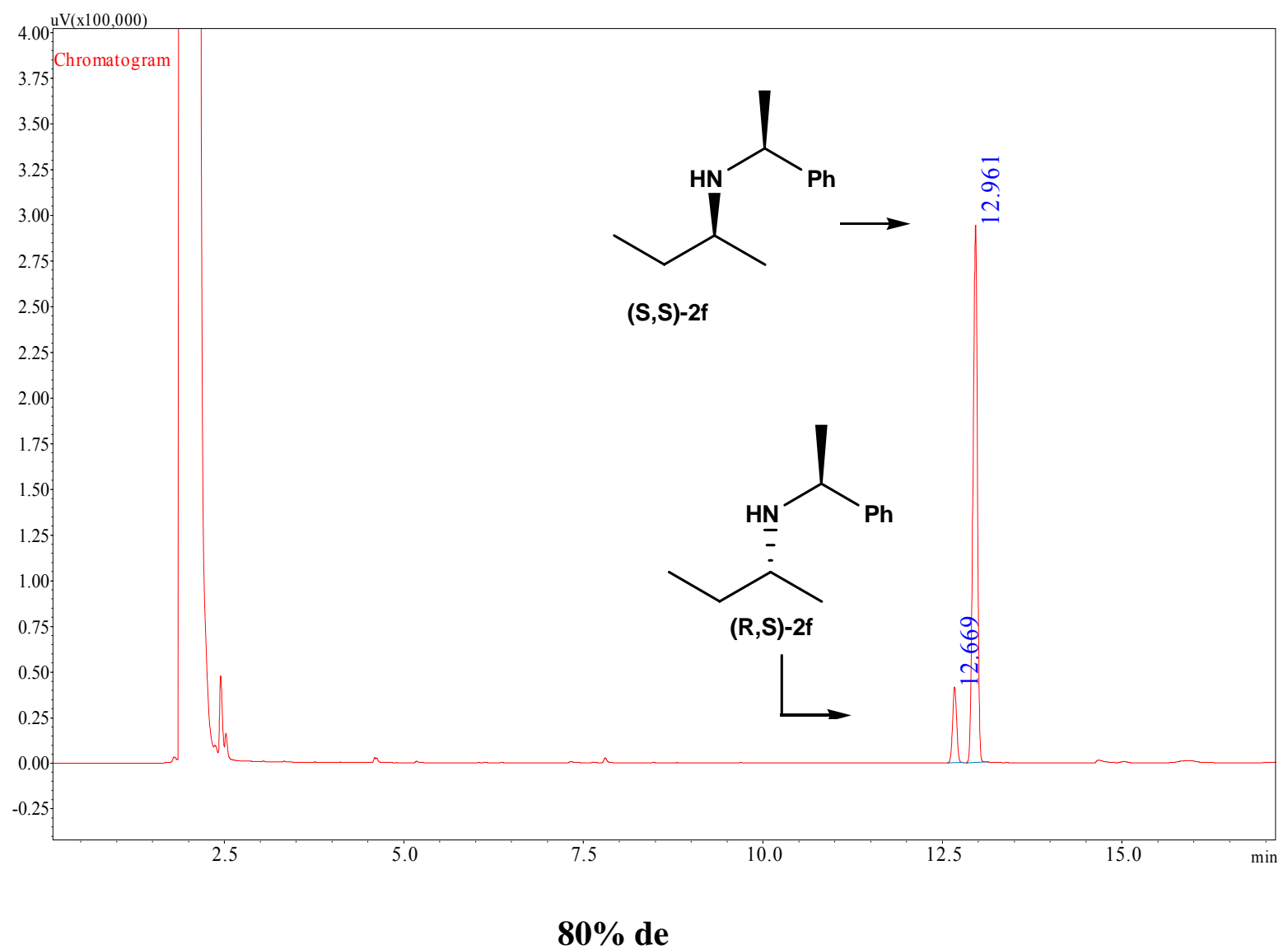

\begin{tabular}{|l|l|l|l|l|}
\hline \multicolumn{5}{|c|}{ Peak Table-Channel 1 } \\
\hline Peak & Ret. Time & Area & Height & Conc. \\
\hline 1 & 12.669 & 185769.5 & 141113.6 & 10.10467 \\
\hline 2 & 12.961 & 1652683.0 & 810635.1 & 89.89533 \\
\hline
\end{tabular}




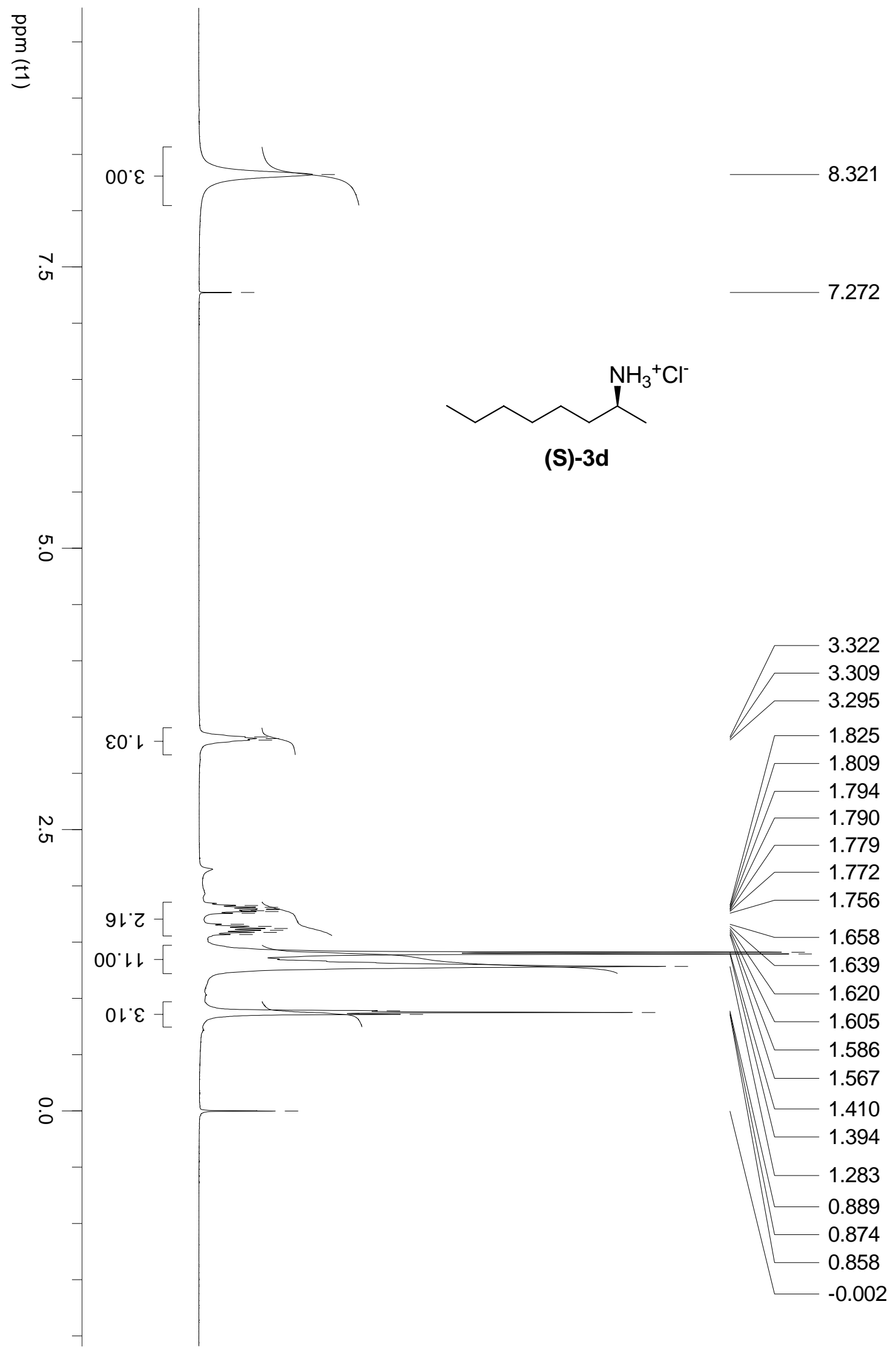




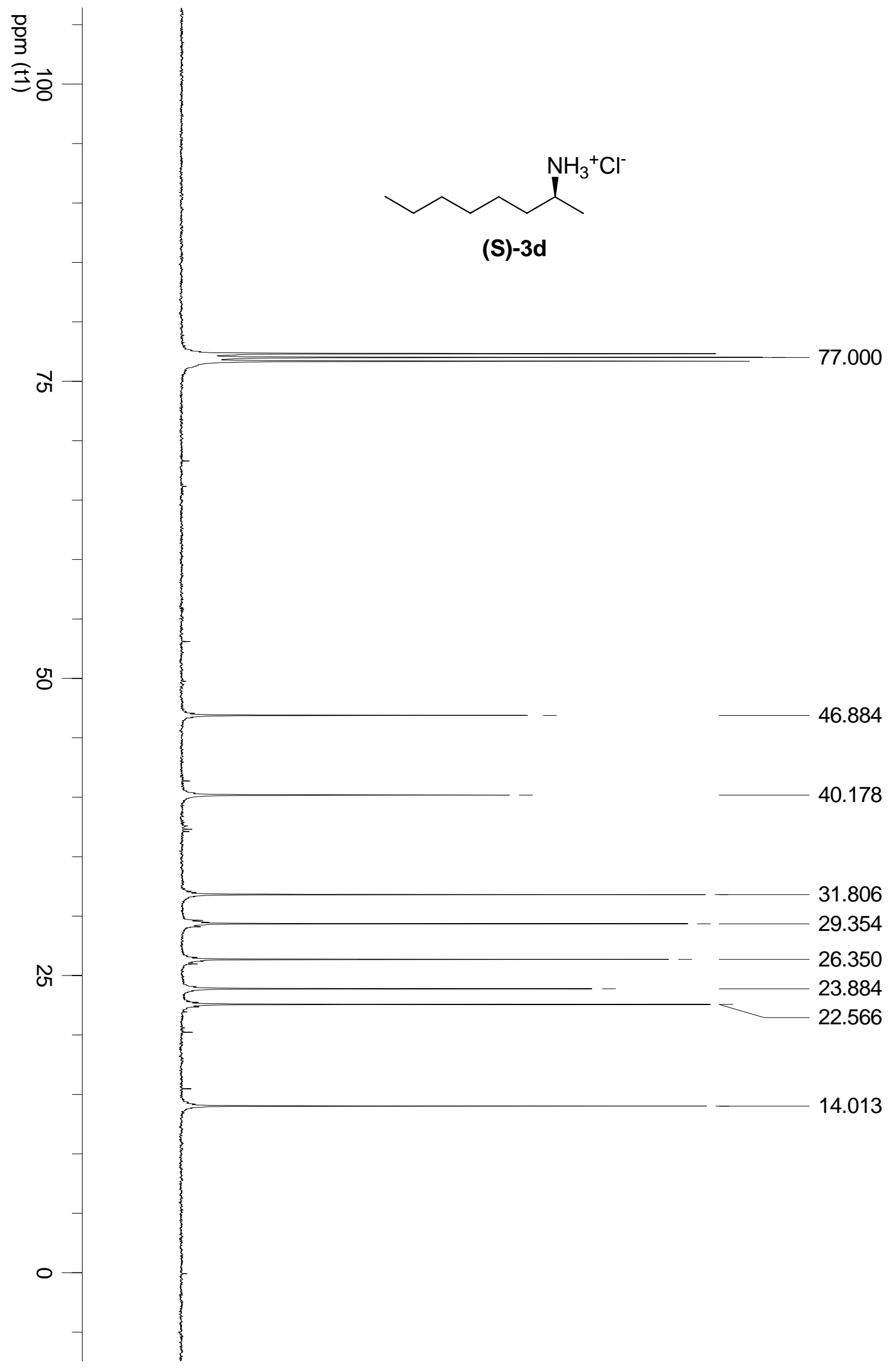




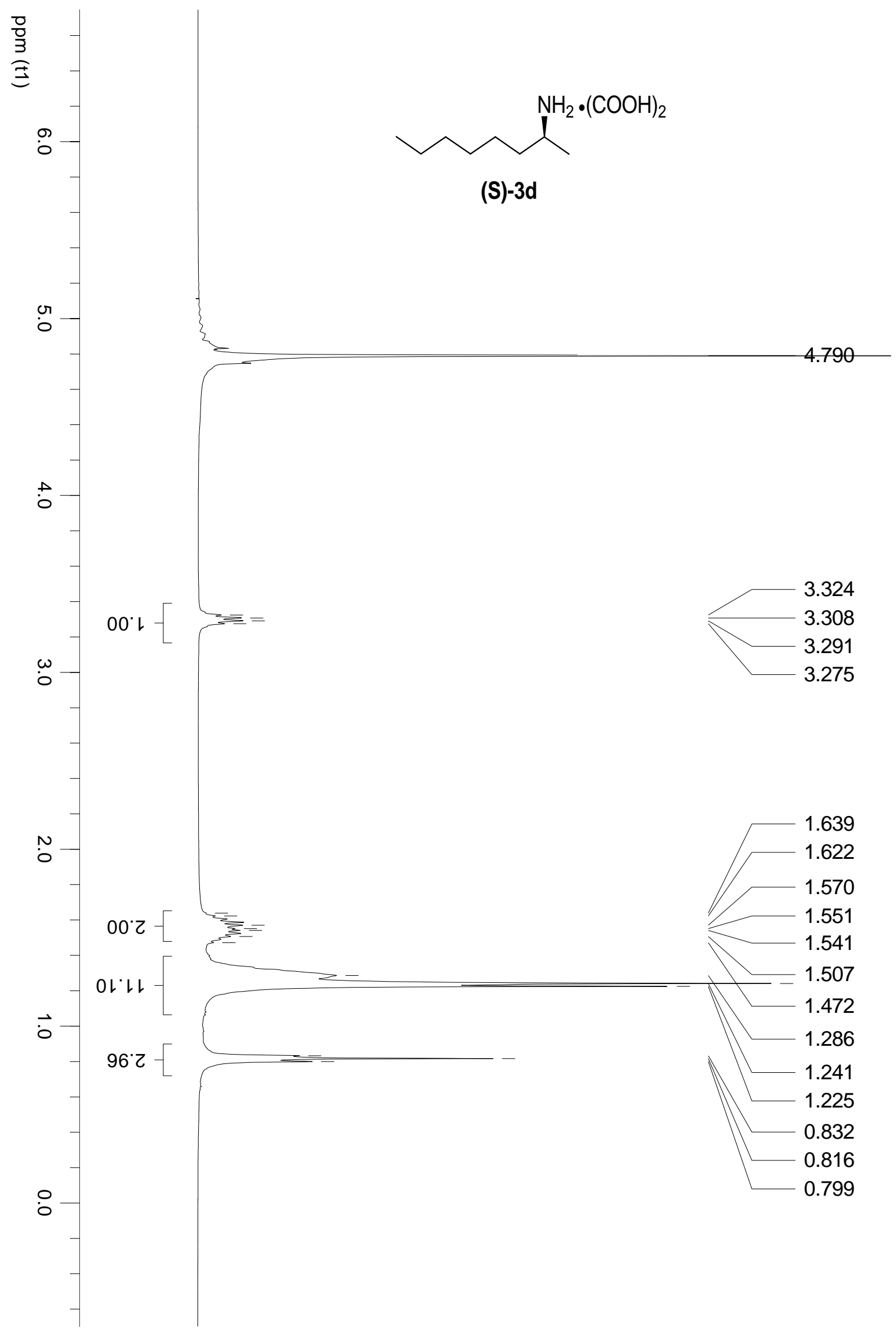




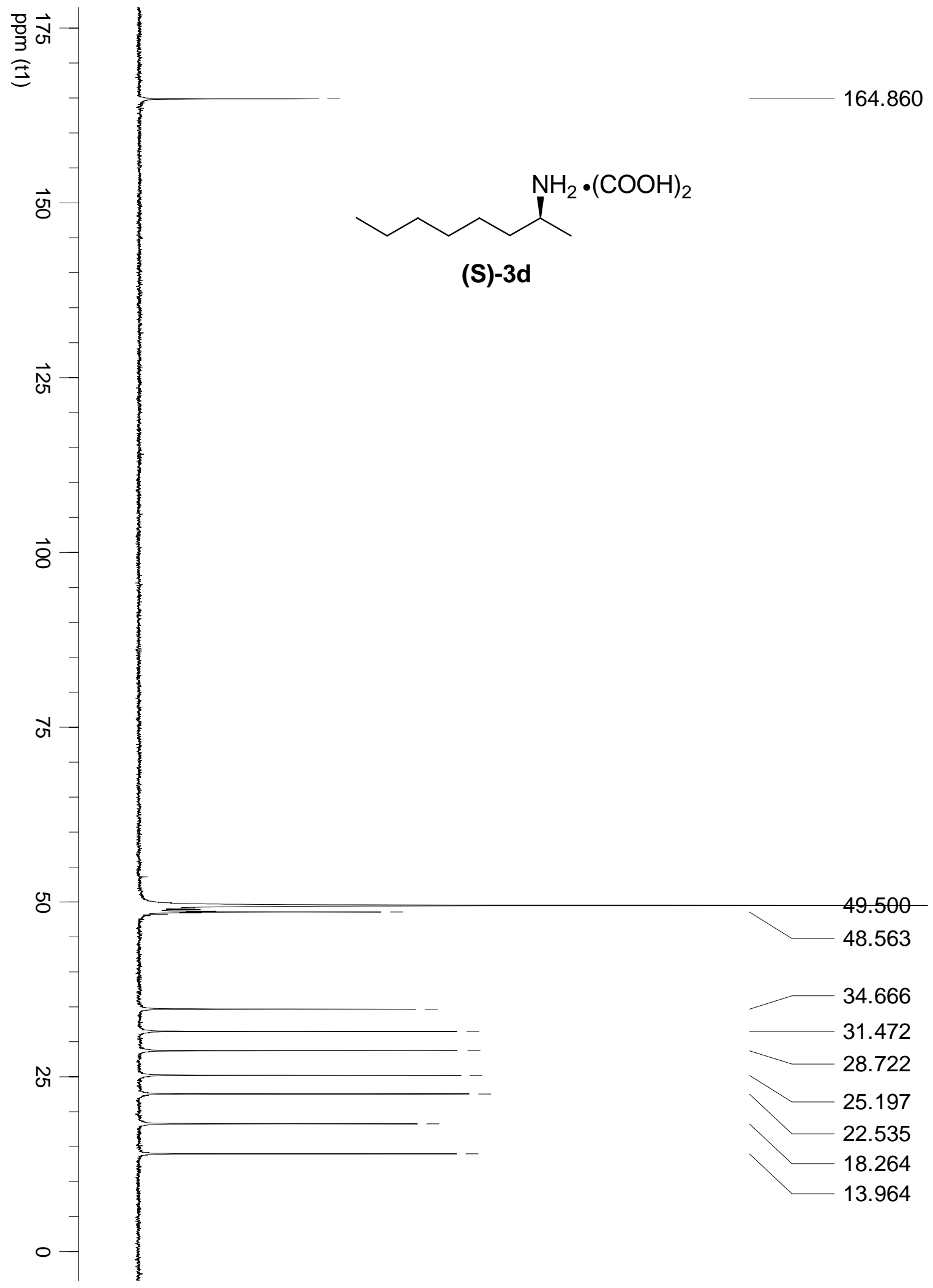




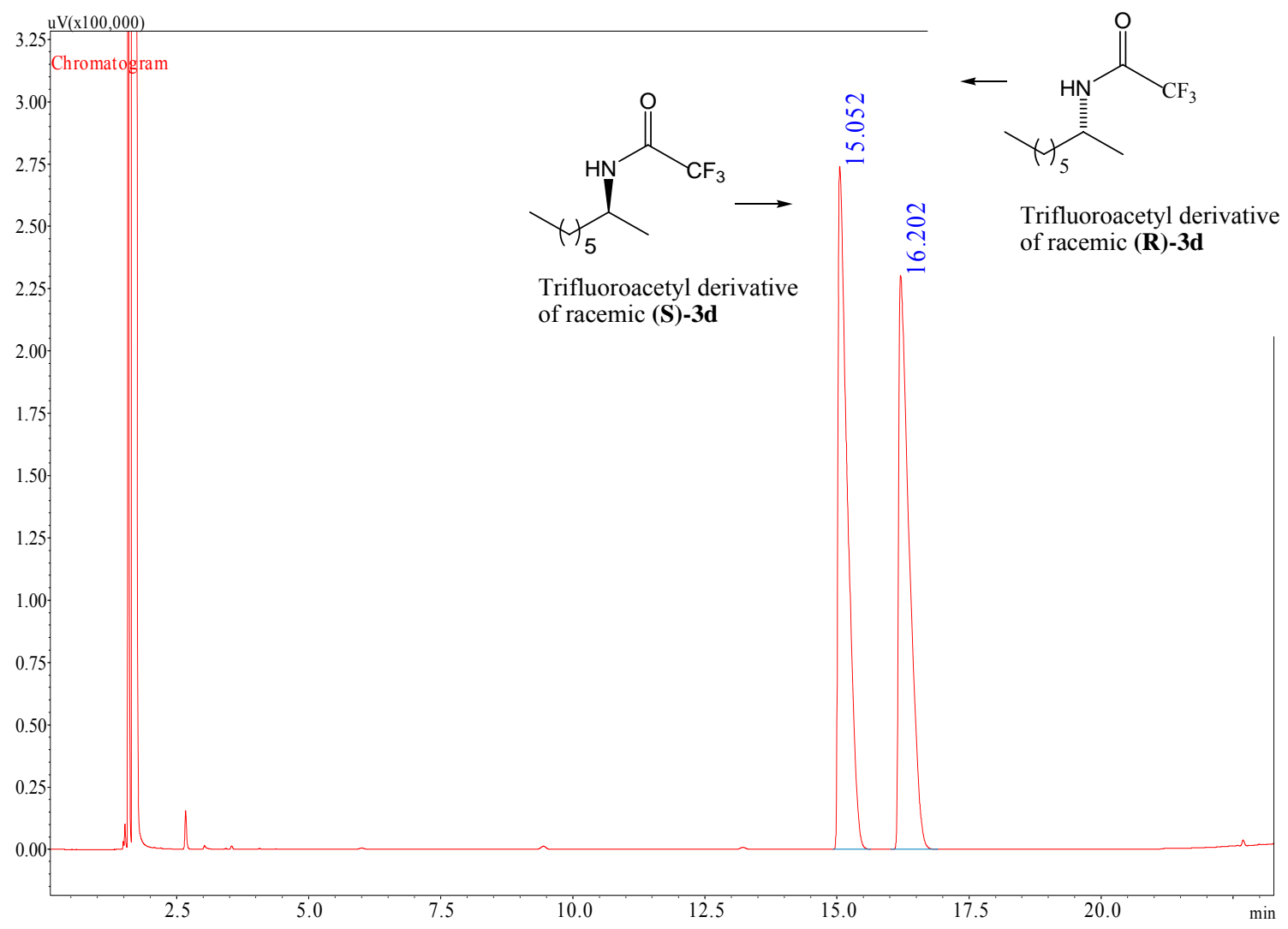

\begin{tabular}{|l|l|l|l|l|}
\hline \multicolumn{5}{|c|}{ Peak Table-Channel 1 } \\
\hline Peak & Ret. Time & Area & Height & Conc. \\
\hline 1 & 15.052 & 3416775.3 & 273965.9 & 51.49583 \\
\hline 2 & 16.202 & 3218276.8 & 230204.8 & 48.50417 \\
\hline
\end{tabular}




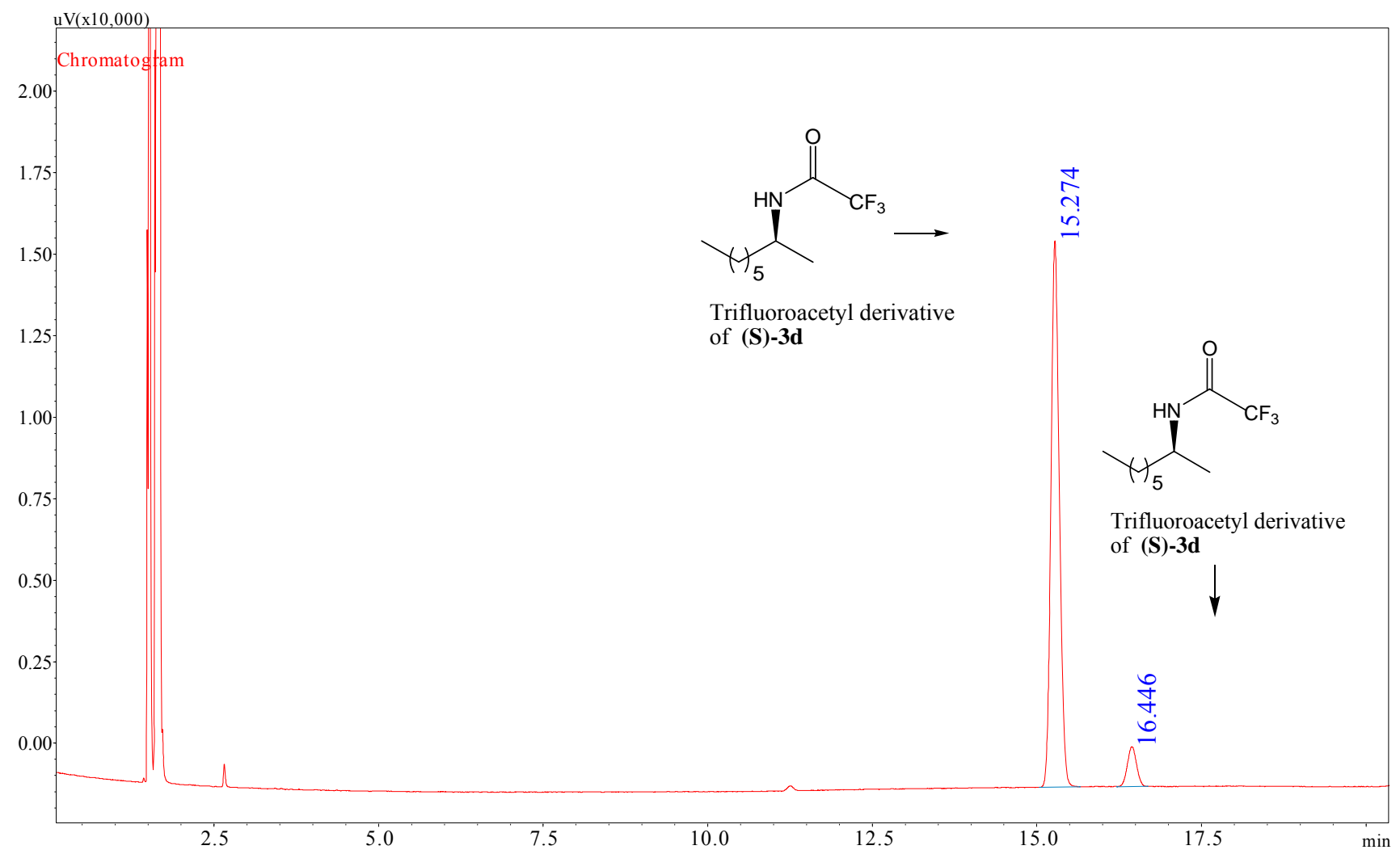

$85 \%$ ee

\begin{tabular}{|l|l|l|l|l|}
\hline \multicolumn{5}{|c|}{ Peak Table-Channel 1 } \\
\hline Peak & Ret. Time & Area & Height & Conc. \\
\hline 1 & 15.274 & 148308.5 & 16752.6 & 92.59243 \\
\hline 2 & 16.446 & 11865.0 & 1217.5 & 7.40757 \\
\hline
\end{tabular}


The gas chromatograms for the catalytic $(10 \mathrm{~mol} \%) \mathrm{Yb}(\mathrm{OAc})_{3}$ promoted asymmetric reductive amination reactions follow. These chromatograms were recorded before washing with a saturated solution of $\mathrm{NH}_{4} \mathrm{Cl}$, thus the compound (S)- $\alpha$ methylbenzylamine is found at retention time $=7-8 \mathrm{~min}$. 


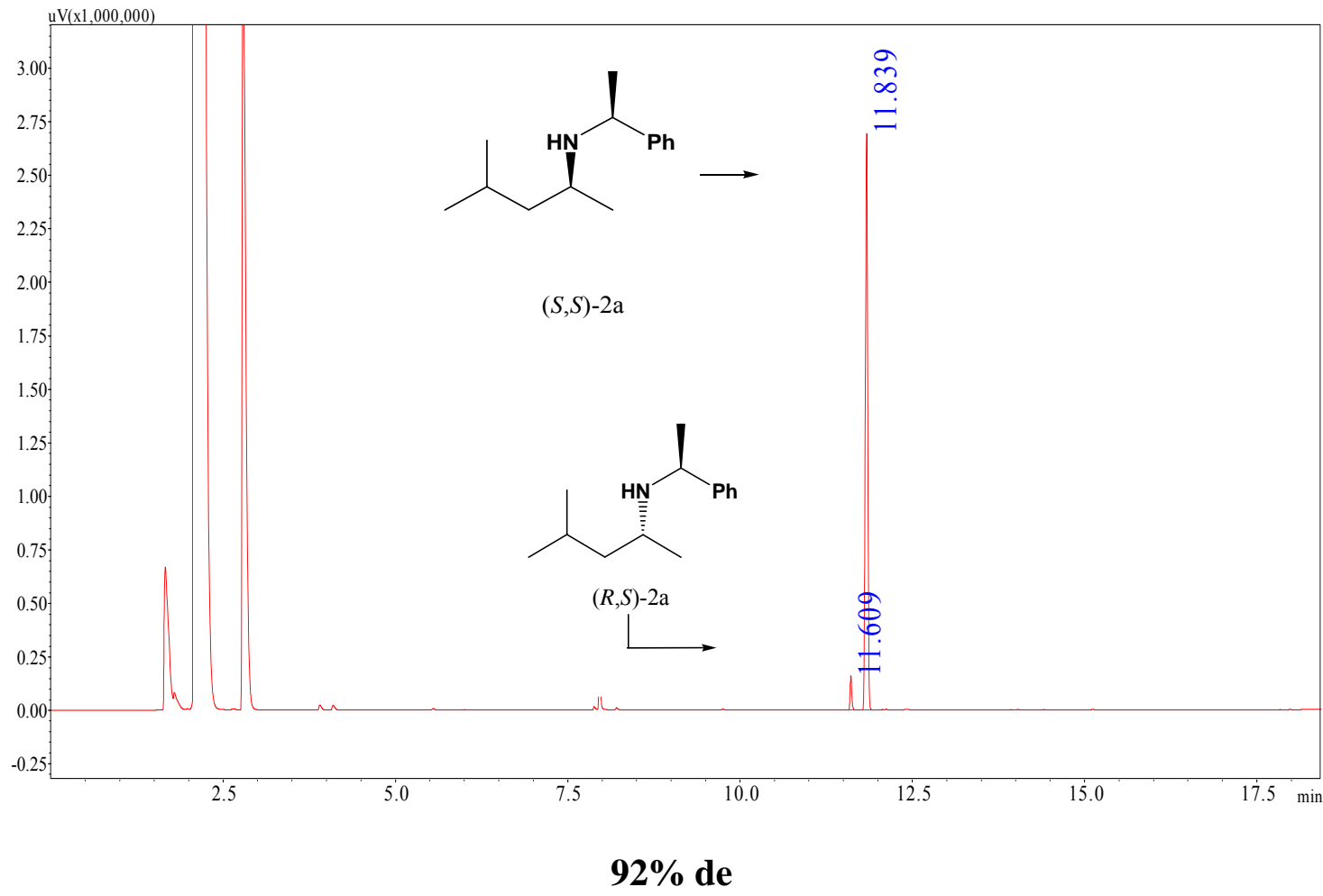

\begin{tabular}{|l|l|l|l|l|}
\hline \multicolumn{5}{|c|}{ Peak Table-Channel 1 } \\
\hline Peak & Ret. Time & Area & Height & Conc. \\
\hline 1 & 11.609 & 267516.0 & 158692.9 & 4.07406 \\
\hline 2 & 11.839 & 6298804.0 & 2677354.6 & 95.92594 \\
\hline
\end{tabular}




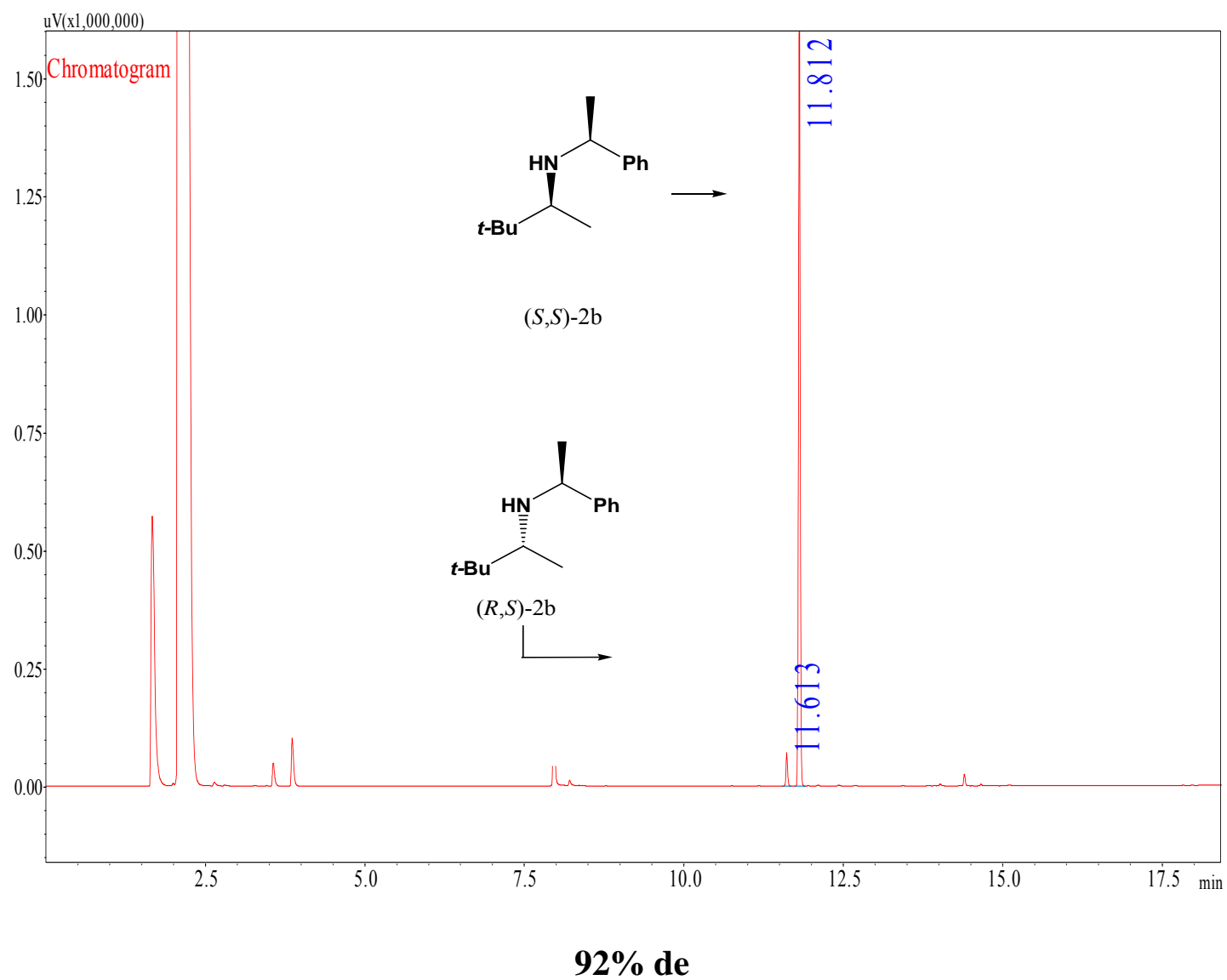

\begin{tabular}{|l|l|l|l|l|}
\hline \multicolumn{5}{|c|}{ Peak Table-Channel 1 } \\
\hline Peak & Ret. Time & Area & Height & Conc. \\
\hline 1 & 11.613 & 120400.3 & 70065.0 & 3.66694 \\
\hline 2 & 11.812 & 3163000.6 & 1731115.5 & 96.33306 \\
\hline
\end{tabular}




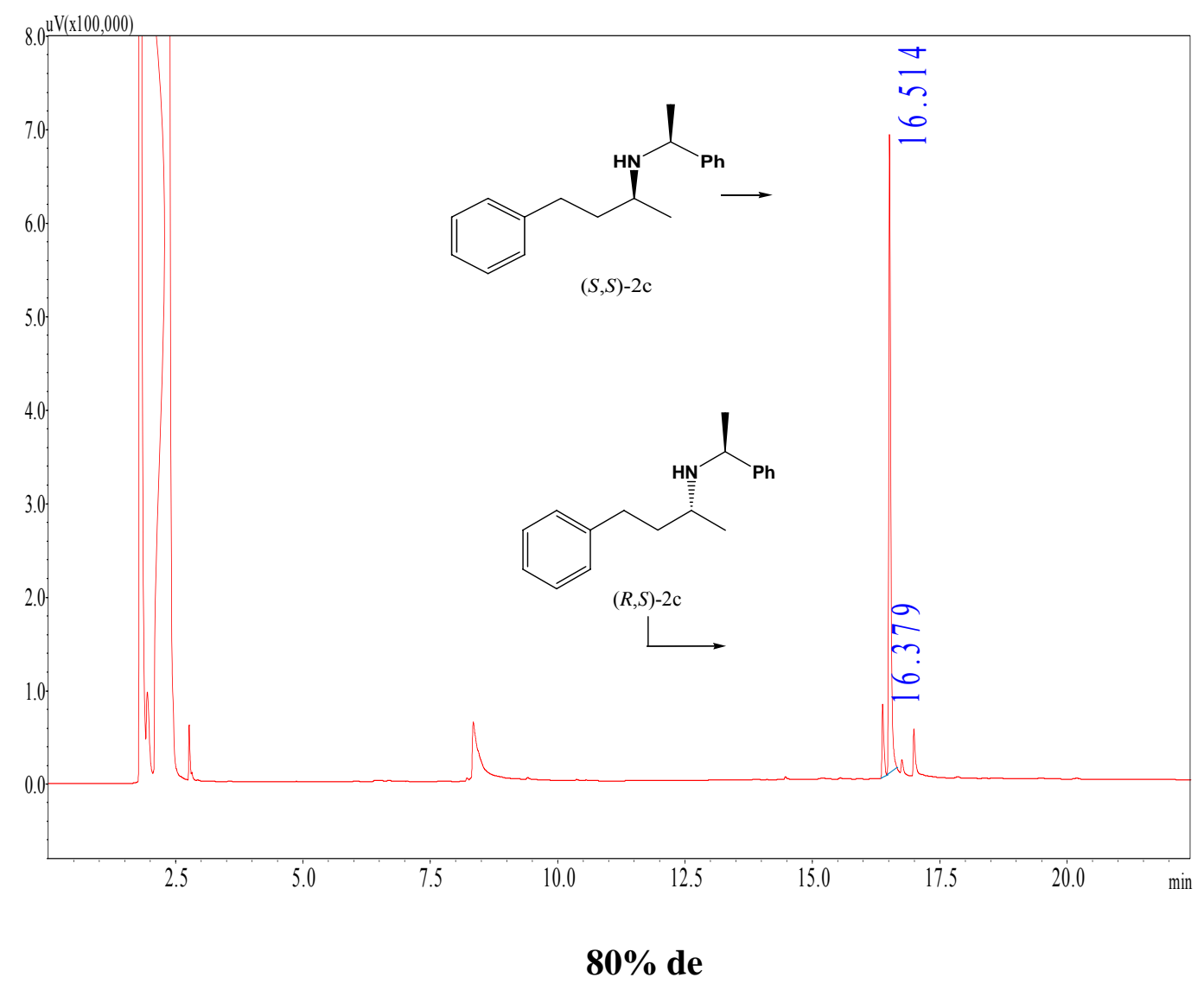

\begin{tabular}{|l|l|l|l|l|}
\hline \multicolumn{5}{|c|}{ Peak Table-Channel 1 } \\
\hline Peak & Ret. Time & Area & Height & Conc. \\
\hline 1 & 16.379 & 199463.6 & 78225.8 & 9.58152 \\
\hline 2 & 16.514 & 1882289.1 & 677685.5 & 90.41848 \\
\hline
\end{tabular}




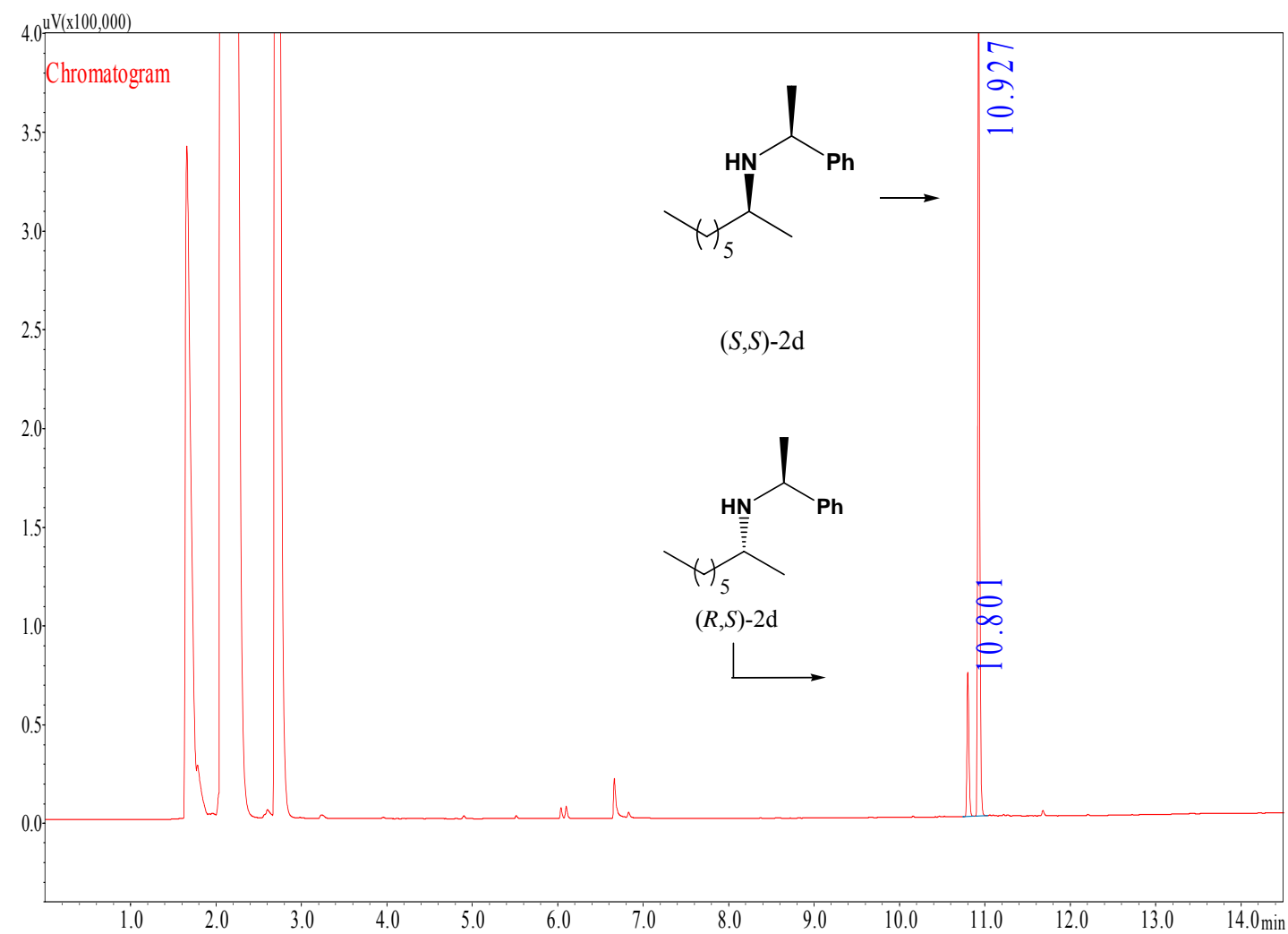

$72 \%$ de

\begin{tabular}{|l|l|l|l|l|}
\hline \multicolumn{5}{|c|}{ Peak Table-Channel 1 } \\
\hline Peak & Ret. Time & Area & Height & Conc. \\
\hline 1 & 10.801 & 109648.4 & 72387.5 & 13.40803 \\
\hline 2 & 10.927 & 708132.8 & 459577.4 & 86.59197 \\
\hline
\end{tabular}




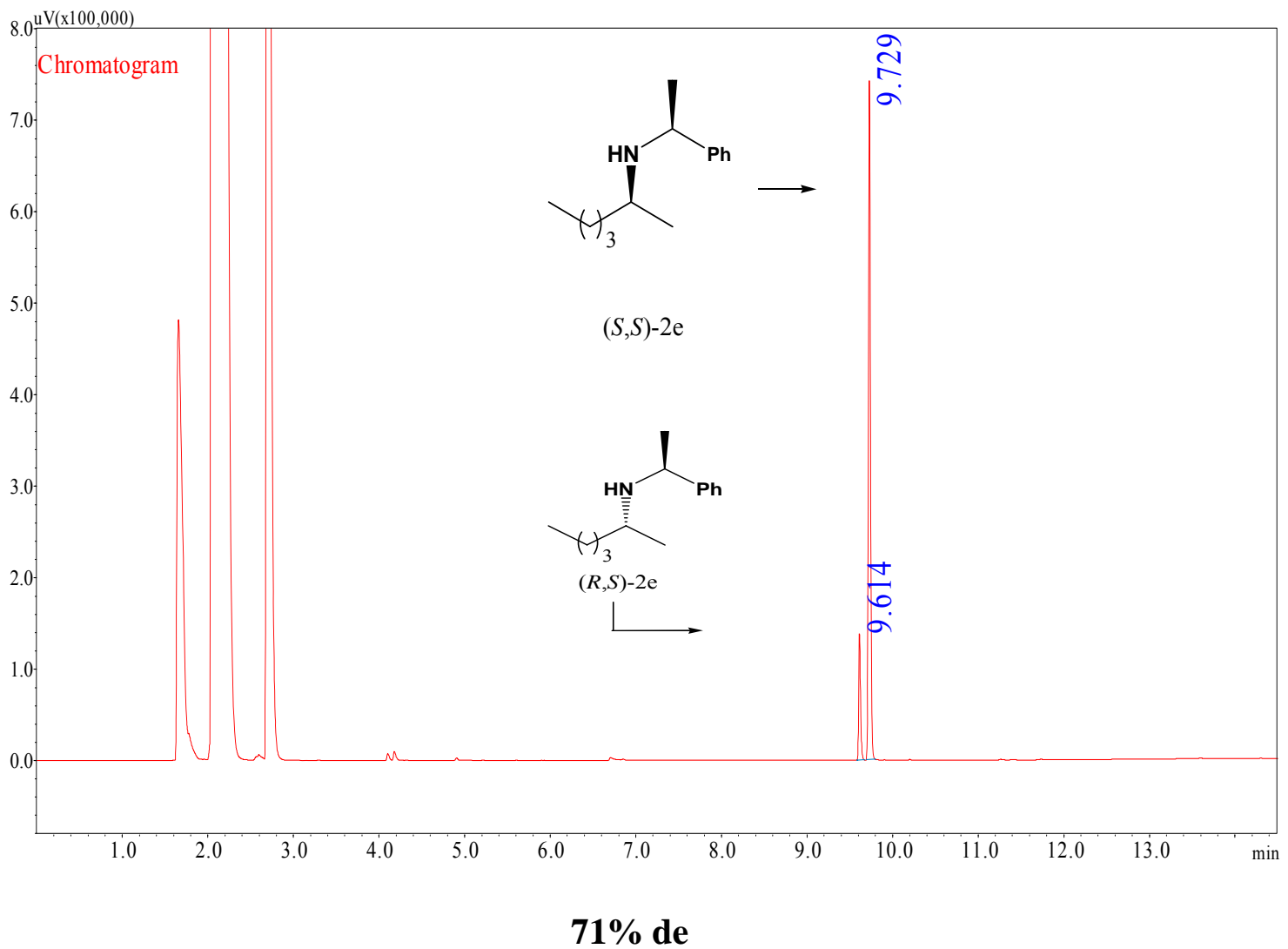

\begin{tabular}{|l|l|l|l|l|}
\hline \multicolumn{5}{|c|}{ Peak Table-Channel 1 } \\
\hline Peak & Ret. Time & Area & Height & Conc. \\
\hline 1 & 9.614 & 205626.5 & 133251.2 & 14.28091 \\
\hline 2 & 9.729 & 1234242.9 & 717510.8 & 85.71909 \\
\hline
\end{tabular}




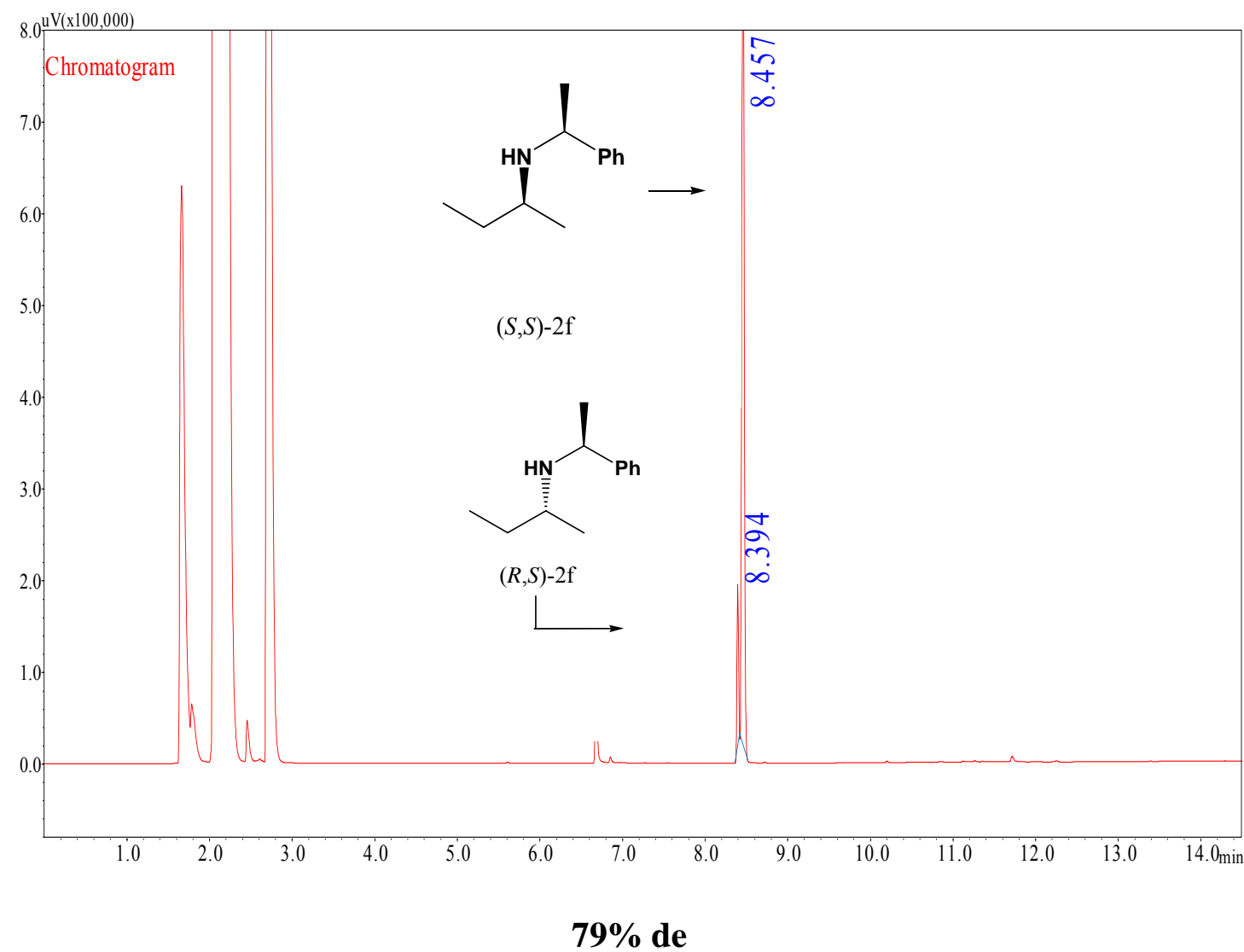

\begin{tabular}{|l|l|l|l|l|}
\hline \multicolumn{5}{|c|}{ Peak Table-Channel 1 } \\
\hline Peak & Ret. Time & Area & Height & Conc. \\
\hline 1 & 8.394 & 226220.2 & 169266.2 & 10.33737 \\
\hline 2 & 8.457 & 1962152.6 & 959367.8 & 89.66263 \\
\hline
\end{tabular}




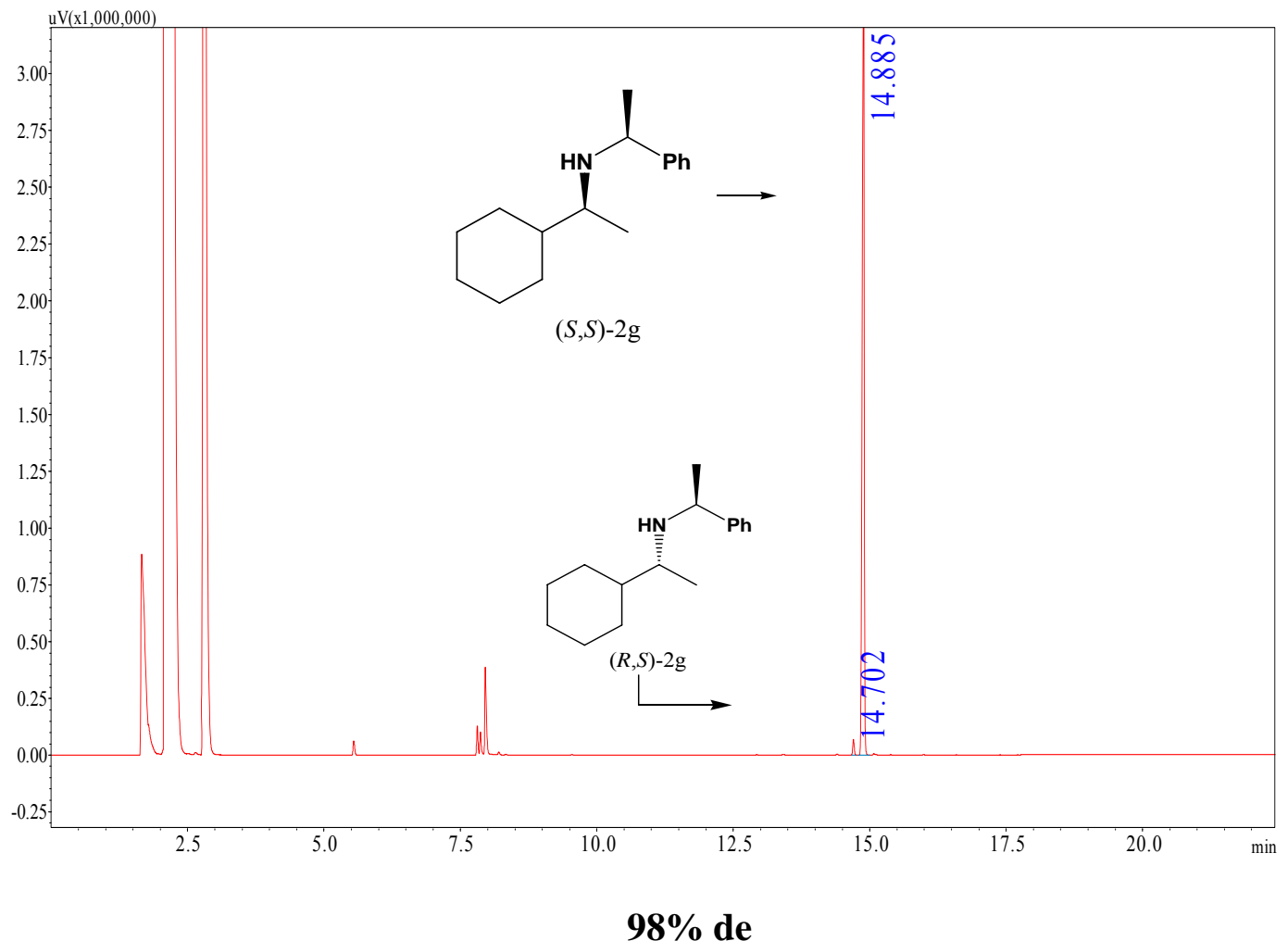

\begin{tabular}{|l|l|l|l|l|}
\hline \multicolumn{5}{|c|}{ Peak Table-Channel 1 } \\
\hline Peak & Ret. Time & Area & Height & Conc. \\
\hline 1 & 14.702 & 121585.6 & 67339.7 & 1.37390 \\
\hline 2 & 14.885 & 8728056.2 & 3796253.7 & 98.62610 \\
\hline
\end{tabular}




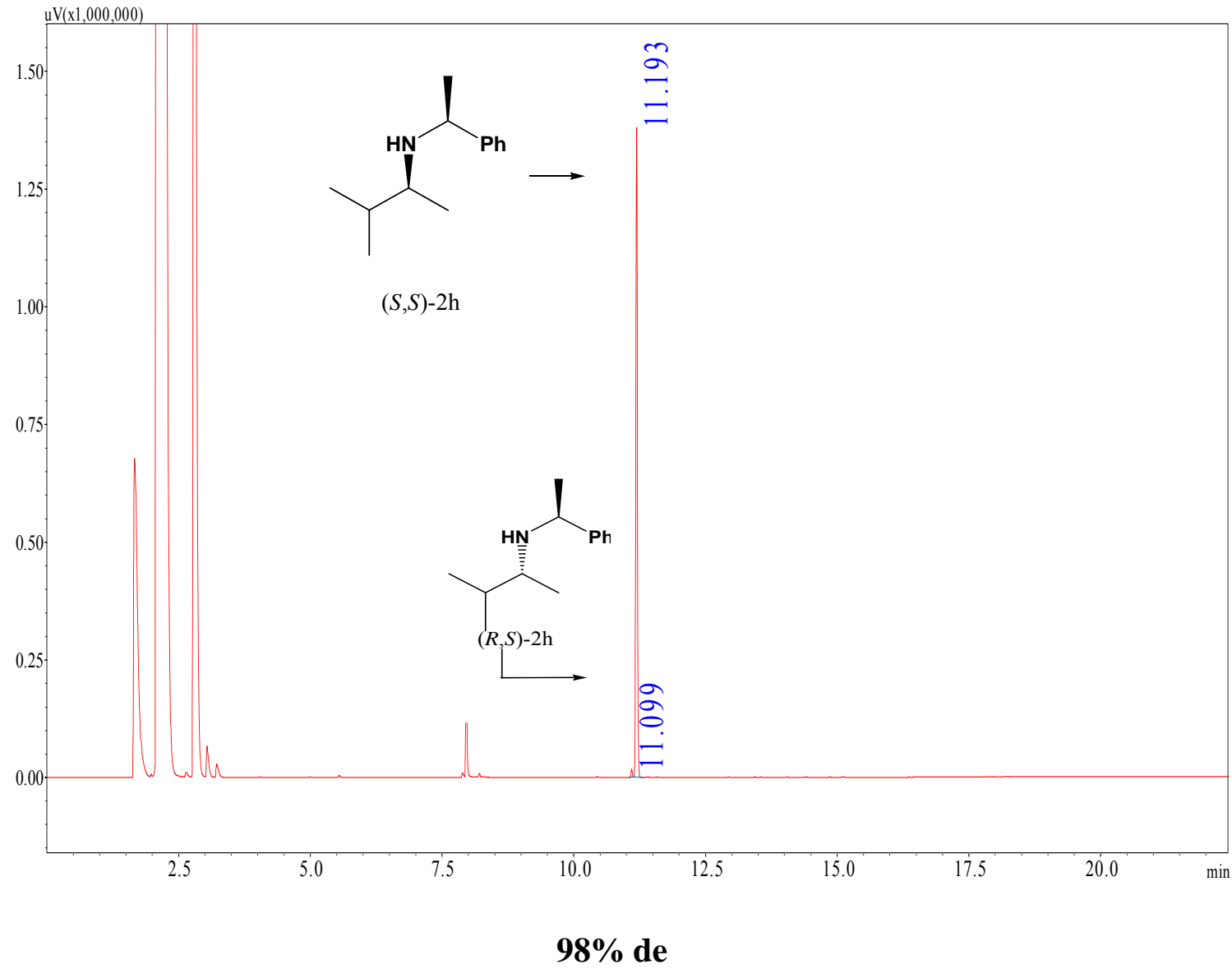

\begin{tabular}{|l|l|l|l|l|}
\hline \multicolumn{5}{|c|}{ Peak Table-Channel 1 } \\
\hline Peak & Ret. Time & Area & Height & Conc. \\
\hline 1 & 11.099 & 29558.6 & 17437.7 & 1.04816 \\
\hline 2 & 11.193 & 2790496.4 & 1374025.9 & 98.95184 \\
\hline
\end{tabular}






94\% de

\begin{tabular}{|l|l|l|l|l|}
\hline \multicolumn{5}{|c|}{ Peak Table-Channel 1 } \\
\hline Peak & Ret. Time & Area & Height & Conc. \\
\hline 1 & 14.401 & 1617391.5 & 880427.8 & 96.51456 \\
\hline 2 & 14.654 & 58409.1 & 33617.6 & 3.48544 \\
\hline
\end{tabular}

